\title{
Photoionization Study of Cl II, Ar II and Kr II Ions Using the Modified Atomic Orbital Theory
}

\author{
Abdourahmane Diallo1, Mamadou Diouldé Ba1, Jean Kouhissoré Badiane, Momar Talla Gning1, \\ Malick Sow ${ }^{2}$, Ibrahima Sakho ${ }^{*}$
}

${ }^{1}$ Department of Physics, UFR Sciences and Technologies, University Assane Seck of Ziguinchor, Ziguinchor, Senegal

${ }^{2}$ Department of Physics, Faculty of Sciences and Techniques, University Cheikh Anta Diop of Dakar, Dakar, Senegal

Email: *aminafatima_sakho@yahoo.fr

How to cite this paper: Diallo, A., Ba, M.D., Badiane, J.K., Gning, M.T., Sow, M. and Sakho, I. (2018) Photoionization Study of $\mathrm{Cl}$ II, Ar II and $\mathrm{Kr}$ II Ions Using the Modified Atomic Orbital Theory. Journal of Modern Physics, 9, 2594-2622. https://doi.org/10.4236/jmp.2018.914162

Received: September 30, 2018

Accepted: December 24, 2018

Published: December 27, 2018

Copyright (๑) 2018 by authors and Scientific Research Publishing Inc. This work is licensed under the Creative Commons Attribution International License (CC BY 4.0).

http://creativecommons.org/licenses/by/4.0/

\section{(c) (i) Open Access}

\begin{abstract}
Resonance energies of the Cl II- $\left[3 \mathrm{~s}^{2} 3 \mathrm{p}^{3}\left({ }^{2} \mathrm{D}_{5 / 2}\right)\right] n d$ and $\left[3 \mathrm{~s}^{2} 3 \mathrm{p}^{3}\left({ }^{2} \mathrm{P}_{3 / 2}\right)\right] n \mathrm{~d}$, Ar II- $3 \mathrm{~s}^{2} 3 \mathrm{p}^{4}\left({ }^{1} \mathrm{D}_{2}\right) n \mathrm{~s}$, $n \mathrm{~d}$ and of the Kr II $\left[4 \mathrm{~s}^{2} 4 \mathrm{p}^{4}\left({ }^{1} \mathrm{D}_{2}\right)\right] n \mathrm{~s}$, nd and $\left.4 \mathrm{~s}^{2} 4 \mathrm{p}^{4}\left({ }^{3} \mathrm{P}_{2},{ }^{3} \mathrm{P}_{1}\right)\right] n \mathrm{~s}$, $\left.4 \mathrm{~s}^{2} 4 \mathrm{p}^{4}\left({ }^{3} \mathrm{D}_{2}\right)\right] n \mathrm{~s}$, nd and $\left.4 \mathrm{~s}^{2} 4 \mathrm{p}^{4}\left({ }^{3} \mathrm{D}_{2},{ }^{1} \mathrm{~S}_{0}\right)\right] n \mathrm{~s}$, nd Rydberg series are reported. Natural widths of the Ar II- $\left[3 \mathrm{~s}^{2} 3 \mathrm{p}^{4}\left({ }^{1} \mathrm{D}_{2}\right)\right] n \mathrm{~s}$, $n$ d series are also reported. Calculations are done in the framework of the Modified Atomic Orbital Theory (MAOT). Excellent agreements are obtained with available theoretical and experimental data. High lying accurate resonance energies up to $n=40$ are tabulated. The possibility to use the MAOT formalism report rapidly with an excellent accuracy the position of the excitation resonances as well as their width within simple analytical formulae is demonstrated.
\end{abstract}

\section{Keywords}

Resonance Energies, Rydberg Series, Natural Widths, Modified Atomic Orbital Theory (MAOT)

\section{Introduction}

In many astrophysical systems such as stars and nebulae, the main process governing light-atomic species interaction is Photoionization. Of great important ions interesting to investigate are $\mathrm{Cl} \mathrm{II}\left(\mathrm{Cl}^{+}\right)$, Ar II $\left(\mathrm{Ar}^{+}\right)$and $\mathrm{Kr}$ II $\left(\mathrm{Kr}^{+}\right)$ions. As far as $\mathrm{Cl}$ II is concerned, its interest is connected with it abundance in photoionized astrophysical objects. In the past, various studies have indicated the great importance of $\mathrm{Cl} \mathrm{II}$ ions abundances for understanding extragalactic HII regions [1]. In addition, emission lines of $\mathrm{Cl} \mathrm{II}$ ions have been observed in the spectra of the Io torus [2] and in the optical spectra of planetary nebulae NGC 6741 and IC 5117 [3]. In a very recent past, Hernández et al., [4] measured at the Advanced Light Source at Lawrence Berkeley National Laboratory absolute photoionization 
cross-sections for the of $\mathrm{Cl}$ II ions using the merged beams photon-ion technique at a photon energy resolution of $15 \mathrm{meV}$ in the energy range $19-28 \mathrm{eV}$. Using the Dirac-Coulomb $R$-matrix (DCR) method, McLaughlin, [5] performed calculations in the same photon energy range that in the ALS experiments [4] to assign and identify the resonance series in the ALS spectra of the Cl II ions. In these experimental works, the $3 s^{2} 3 \mathrm{p}^{3} n$ d states have been identified in the $\mathrm{Cl}$ II spectra as the prominent Rydberg series belonging to the $3 \mathrm{p} \rightarrow n$ d transitions. The weaker $3 s^{2} 3 p^{3} n$ s Rydberg series, identified as $3 p \rightarrow n$ s transitions and window resonances $3 \mathrm{~s} 3 \mathrm{p}^{4}\left({ }^{4} \mathrm{P}\right) n \mathrm{p}$ features, due to $3 \mathrm{~s} \rightarrow n \mathrm{p}$ transitions, have also been found in the spectra [5]. Besides, one of the important elements to study is Argon present in several astrophysical systems. An overabundance of the argon element in the spectra of X-rays of yellow supernovas was revealed by the satellite Chandra [6] dedicated to analyze the stellar object spectra. Furthermore, spectral rays of the argon element were observed in the optical spectra of planetary stars and nebulae [7] [8]. These few examples show the importance of the photoionization study of the argon element from the perspective of astrophysics. In a recent past, Covington et al., [9] performed the first experimental measurements of the photoionization cross-section of the Ar II ion. These authors also determined the resonance energies and natural widths related to the dominant Rydberg series $3 \mathrm{~s}^{2} 3 \mathrm{p}^{4}\left({ }^{1} \mathrm{D}_{2}\right) n \mathrm{~s}$, nd and $3 \mathrm{~s}^{2} 3 \mathrm{p}^{4}\left({ }^{1} \mathrm{~S}_{0}\right) n \mathrm{~s}$, nd in the emission spectra of the Ar II ions. These energies were relatively measured at the metastable state Ar II $\left({ }^{2} \mathrm{P}_{1 / 2}^{\circ}\right)$ and at the ground state Ar II $\left({ }^{2} \mathrm{P}_{3 / 2}^{\circ}\right)$. As far as Krypton is concerned, it is also an element of major importance for diagnosing stellar plasmas such as stars and planetary nebulae as well as for diagnosing laboratory plasmas such as those obtained by inertial fusion. In a recent past, Hinojosa et al., [10] experimentally studied the photoionization of the Kr II ion at ALS at Berkley in the photonic energy range of $23-39 \mathrm{eV}$. In the photoionization spectra, these authors observed several Rydberg series, including the Kr II $\left[4 s^{2} 4 \mathrm{p}^{4}\left({ }^{1} \mathrm{D}_{2}\right)\right] n s$, nd series converging toward the excitation threshold $\mathrm{Kr}$ II $\left[4 \mathrm{~s}^{2} 4 \mathrm{p}^{4}\left({ }^{1} \mathrm{D}_{2}\right)\right]$. Very recently, Sakho, [11] applied the Screening constant by unit nuclear charge (SCUNC) formalism to report precise data belonging to various Rydberg series of Ar II and $\mathrm{Kr}$ II as observed in the works of Covington et al., [9] for Ar II and of Hinojosa et al., [10] for Kr II. In the present study, we use the Modified atomic orbital theory [12] [13] [14] [15] [16] to report accurate high lying resonance energies of the Cl I- $\left[3 \mathrm{~s}^{2} 3 \mathrm{p}^{3}\left({ }^{2} \mathrm{D}_{5 / 2}\right)\right] n d$ and $\left[3 \mathrm{~s}^{2} 3 \mathrm{p}^{3}\left({ }^{2} \mathrm{P}_{3 / 2}\right)\right] n d$, Ar II- $3 \mathrm{~s}^{2} 3 \mathrm{p}^{4}\left({ }^{1} \mathrm{D}_{2}\right) n s$, nd and of the Kr II $\left[4 \mathrm{~s}^{2} 4 \mathrm{p}^{4}\left({ }^{1} \mathrm{D}_{2}\right)\right] n s$, nd and $\left.\left.4 \mathrm{~s}^{2} 4 \mathrm{p}^{4}\left({ }^{3} \mathrm{P}_{2},{ }^{3} \mathrm{P}_{1}\right)\right] n \mathrm{~s}, 4 \mathrm{~s}^{2} 4 \mathrm{p}^{4}\left({ }^{3} \mathrm{D}_{2}\right)\right] n s$, $n d$ and $\left.4 \mathrm{~s}^{2} 4 \mathrm{p}^{4}\left({ }^{3} \mathrm{D}_{2},{ }^{1} \mathrm{~S}_{0}\right)\right] n s$, nd Rydberg series reported. Natural widths of the Ar II- $\left[3 s^{2} 3 \mathrm{p}^{4}\left({ }^{1} \mathrm{D}_{2}\right)\right] n s, n d$ series are also reported. In Section 2, a brief description of the MAOT formalism is given. The results are present in Section 3. Section 4 concludes the study.

\section{Theory}

\subsection{Brief Description of the Modified Atomic Orbital Theory}

In the framework of the modified atomic orbital theory (MAOT), the total 
energy of a $(v l)$-given orbital is expressed in the form in Rydberg units

$$
E(v l)=-\frac{[Z-\sigma(l)]^{2}}{v^{2}}
$$

In Equation (1), $\sigma$ is the screening constant relative to the electron occupying the $(v l)$-orbital, $l$ denotes the orbital quantum number, $v$ stands for the principal quantum number and $Z$ represents the atomic number. In general, the doubly excited states (DES) in two electron systems are labelled as $\left(N l, n l^{\prime}\right)^{2 S+1} L^{\pi}$. In this notation, $N$ and $n$ denote respectively the principal quantum numbers of the inner and the outer electron, $I$ and $I$ 'are their respective orbital quantum numbers, $S$ the total spin, $L$ the total angular momentum and $\pi$ the parity of the system. For an atomic system of many $M$ electrons, total energy is expressed as follows

$$
E=-\sum_{i=1}^{M} \frac{\left[Z-\sigma_{i}\left({ }^{2 S+1} L^{\pi}\right)\right]^{2}}{v_{i}^{2}}
$$

In the photoionisation study, energy resonances $E_{n}$ are generally measured relatively to the $E_{\infty}$ converging limit of a given $\left({ }^{2 S+1} L_{J}\right) n l$-Rydberg series. For these states [12] [13] [14]

$$
\begin{aligned}
E_{n}= & E_{\infty}-\frac{1}{n^{2}}\left\{Z-\sigma_{1}\left({ }^{2 S+1} L_{J}\right)-\sigma_{2}\left({ }^{2 S+1} L_{J}\right) \times \frac{1}{n}\right. \\
& \left.-\sigma_{2}^{\alpha}\left({ }^{2} P_{3 / 2}^{0},{ }^{1} D_{2}\right) \times(n-m) \times(n-q) \sum_{k} \frac{1}{f_{k}(n, m, q, s)}\right\}^{2}
\end{aligned}
$$

In this equation, $m$ and $q(m<q)$ denote the principal quantum numbers of the $\left({ }^{2 S+1} L_{J}\right) n l$-Rydberg series of the considered atomic system used in the empirical determination of the $\sigma_{i}\left({ }^{2 S+1} L_{J}\right)$-screening constants, $s$ represents the spin of the nl-electron $(s=1 / 2), E_{\infty}$ is the energy value of the series limit generally determined from NIST atomic database, and $Z$ represents the nuclear charge of the considered element. The only problem that one may face by using the MAOT formalism is linked to the determination of the $\sum_{k} \frac{1}{f_{k}(n, m, q, s)}$-term. The correct expression of this term is determined iteratively by imposing general Equation (3) to give accurate data with a constant quantum defect values along all the considered series. The value of $\alpha$ is fixed to 1 and or 2 during the iteration. The quantum defect is calculated from the standard formula

$$
E_{n}=E_{\infty}-\frac{R Z_{\text {core }}^{2}}{(n-\delta)^{2}}
$$

In this equation, $R$ is the Rydberg constant, $E_{\infty}$ denotes the converging limit, $Z_{\text {core }}$ represents the electric charge of the core ion, and $\delta$ means the quantum defect. As far as the natural widths are concerned, they are given by (in Rydberg units) 


$$
\begin{aligned}
\Gamma_{n}= & \frac{1}{n^{2}}\left\{Z-\sigma_{1}^{\prime}\left({ }^{2 S+1} L_{J}\right)-\sigma_{2}^{\prime}\left({ }^{2 S+1} L_{J}\right) \times \frac{1}{n}\right. \\
& \left.-\sigma_{2}^{\alpha^{\prime}}\left({ }^{2} P_{3 / 2}^{0},{ }^{1} D_{2}\right) \times(n-m) \times(n-q) \sum_{k} \frac{1}{f_{k}^{\prime}(n, m, q, s)}\right\}^{2}
\end{aligned}
$$

\subsection{Expressions of the Resonance Energies and of the Natural Widths}

In the present work, for all the Rydberg series investigated for both Cl II, Ar II and $\mathrm{Kr}$ II, the resonance energies are given by the formula

$$
\begin{aligned}
E_{n}= & E_{\infty}-\frac{1}{n^{2}}\left\{Z-\sigma_{1}\left({ }^{2 S+1} L_{J}\right)-\sigma_{2}\left({ }^{2 S+1} L_{J}\right) \times \frac{1}{n}\right. \\
& -\sigma_{2}^{2}\left({ }^{2 S+1} L_{J}\right) \times(n-m)^{2}\left[\frac{1}{(n+s+2)^{4}}+\frac{1}{(n-2 s)^{4}}\right] \\
& \left.+\sigma_{2}\left({ }^{2 S+1} L_{J}\right) \times(n-m)^{2}\left[\frac{1}{(n+m+s)^{4}}+\frac{1}{(n+m-s)^{4}}\right]\right\}^{2}
\end{aligned}
$$

For the $\left[3 \mathrm{~s}^{2} 3 \mathrm{p}^{4}\left({ }^{1} \mathrm{D}_{2}\right)\right] n \mathrm{~s}(j=1 / 2)$ series originating from the $3 \mathrm{~s}^{2} 3 \mathrm{p}^{5} \mathrm{P}_{1 / 2}^{0}$ metastable state of Ar II ions, the natural widths are given by (in Rydberg units)

$$
\begin{aligned}
\Gamma_{n}= & \frac{1}{n^{2}}\left\{Z-\sigma_{1}\left({ }^{2 S+1} L_{J}\right)-\sigma_{2}\left({ }^{2 S+1} L_{J}\right) \times \frac{1}{n}+\sigma_{2}^{2}\left({ }^{2 S+1} L_{J}\right) \times(n-m) \times(n-q)\right. \\
& \left.\times\left[\frac{1}{(n+q-m+s+1)^{4}}+\frac{1}{(n+m+s)^{4}}+\frac{1}{(n+q+s)^{4}}+\frac{1}{(n+m-s)^{4}}\right]\right\}^{2}
\end{aligned}
$$

The other expressions for the other series are of type Equation (7).

\section{Results and Discussion}

The $\sigma_{1}$-screening constants in Equations (6) and (7) are evaluated empirically using the data from Covington et al., [9] Hinojosa et al., [10] and from Hernández et al., [4]. The results obtained as indicated in the caption of the corresponding Table. As far as the $\sigma_{2}$-screening constant is concerned, it is evaluated theoretical from the simple equation $\sigma_{2}=Z-Z_{\text {core. }}$ The electric charge of the core ion is deduced directly from the single Photoionization process for a given $\mathrm{X}^{\mathrm{p}+}$-plasma ion

$$
h v+\mathrm{X}^{\mathrm{p}+} \rightarrow \mathrm{X}^{(\mathrm{p}+1)}+\mathrm{e}^{-} \Rightarrow Z_{\text {core }}=(\mathrm{p}+1)
$$

So, for $\mathrm{Cl}$ II, Ar II and $\mathrm{Kr}$ II, we find respectively.

$$
\begin{aligned}
& h v+\mathrm{Cl}^{+} \rightarrow \mathrm{Cl}^{2+}+\mathrm{e}^{-} ; Z_{\text {core }}=2 \Rightarrow \sigma_{2}=15.00 \\
& h v+\mathrm{Ar}^{+} \rightarrow \mathrm{Ar}^{2+}+\mathrm{e}^{-} ; Z_{\text {core }}=2 \Rightarrow \sigma_{2}=16.00 \\
& h v+\mathrm{Kr}^{+} \rightarrow \mathrm{Kr}^{2+}+\mathrm{e}^{-} ; Z_{\text {core }}=2 \Rightarrow \sigma_{2}=34.00
\end{aligned}
$$

The resonance energies of the $\left[3 \mathrm{~s}^{2} 3 \mathrm{p}^{3}\left({ }^{2} \mathrm{D}_{5 / 2}^{0}\right)\right]$ nd Rydberg series originating from the $3 s^{2} 3 p^{43} \mathrm{P}_{2}^{0}$ ground state and from the $3 s^{2} 3 p^{43} \mathrm{P}_{1}^{\circ}, 3 s^{2} 3 p^{43} \mathrm{P}_{0}^{0}$, 
$3 \mathrm{~s}^{2} 3 \mathrm{p}^{4} \mathrm{~S}_{0}^{\circ}$ and $3 \mathrm{~s}^{2} 3 \mathrm{p}^{41} \mathrm{D}_{2}$ metastable states of $\mathrm{Cl}$ II ions are listed in Tables 1-8. Comparisons of the present MAOT calculations are done with the available Dirac-Coulomb $R$-matrix (DCR) calculations [5] and with the ALS experimental data [4]. For both the DCR [5] and ALS [4] studies, the determination of the resonances energies have been limited to $n=13$ (see Table 4). In general due to interaction configuration and other electron-electron effects, the peaks of the cross section overlap involving difficulty for the identification of lines in the atomic spectra with increasing $n$. But, it can be seen that, the MAOT formulas are enough stable so that very high lying resonances can be tabulate up to $n=40$ with a quantum defect practically constant along all the series investigated. For many resonances, the uncertain experimental entries in parenthesis are enlightened. In Table 2, the resonance energy of the $\left[3 \mathrm{~s}^{2} 3 \mathrm{p}^{3}\left({ }^{2} \mathrm{D}_{5 / 2}^{0}\right)\right] 11 \mathrm{~d}$ level are equal to $25.493 \mathrm{eV}$ (MAOT), $25.493 \mathrm{eV}$ (DCR) and (25.492 eV) for the ALS uncertain experimental data. The excellent agreement between theories indicate that the ALS data can be stated as accurate at $25.492 \mathrm{eV}$. The same conclusion can be drawn for the $\left[3 \mathrm{~s}^{2} 3 \mathrm{p}^{3}\left({ }^{2} \mathrm{D}_{5 / 2}^{\circ}\right)\right] 8 \mathrm{~d}$ state quoted in Table 3 where the MAOT prediction at $25.001 \mathrm{eV}$ agree very well with both the DCR value at $24.999 \mathrm{eV}$ [5] and the uncertain ALS measurement [4] equal to (25.000 eV). For this level the ALS data must be considered as precise at $25.000 \mathrm{eV}$. Table 4 lists resonance energies of the $\left[3 \mathrm{~s}^{2} 3 \mathrm{p}^{3}\left({ }^{2} \mathrm{P}_{3 / 2}^{\circ}\right)\right] n d$ Rydberg series originating from the $3 \mathrm{~s}^{2} 3 \mathrm{p}^{4} \mathrm{P}_{2}^{\circ}$ ground state of the $\mathrm{Cl}^{+}$ions. In this table, two uncertain ALS values are quoted for the $\left[3 s^{2} 3 p^{3}\left({ }^{2} P_{3 / 2}^{0}\right)\right] 6 d$ and $\left[3 s^{2} 3 p^{3}\left({ }^{2} P_{3 / 2}^{0}\right)\right] 12 d$ levels respectively at $(25.745 \mathrm{eV})$ and $(27.114 \mathrm{eV})$ to be compared to the MAOT predictions at $25.749 \mathrm{eV}$ and $27.113 \mathrm{eV}$ and to the DCR data [5] respectively equal to $25.755 \mathrm{eV}$ and $27.115 \mathrm{eV}$. For the $n=11$ and 13, the MAOT calculations respectively at $27.031 \mathrm{eV}$ and $27.176 \mathrm{eV}$ are seen to agree very well with the ALS measurements [4] at $27.031 \mathrm{eV}$ and $27.175 \mathrm{eV}$. Subsequently the DCR data at 25.755 $\mathrm{eV}(n=6)$ and at $27.178 \mathrm{eV}(n=13)$ are probably greater than the accurate data. The ALS experimental entries in parenthesis can be considered as certain at $25.745 \mathrm{eV}$ and $27.114 \mathrm{eV}$. Besides, the MAOT data at $25.659 \mathrm{eV}$ quoted in Table 5 is seen to agree very well with the uncertain ALS measurement [4] at (25.660 $\mathrm{eV})$. A slight discrepancy is observed when comparing with the corresponding DCR calculation [5] equal to $25.668 \mathrm{eV}$. Comparison indicates clearly that the ALS value [4] is correct at $25.660 \mathrm{eV}$. In Table 6 and Table 7, all the ALS data [4] are certain. In general, good agreements are obtained between theory and experiment. In Table 8, the uncertain ALS data [4] at $(24.152 \mathrm{eV})$ for the $\left[3 \mathrm{~s}^{2} 3 \mathrm{p}^{3}\left({ }^{2} \mathrm{D}_{5 / 2}^{\circ}\right)\right] 11 \mathrm{~d}$ level is difficult to enlighten. For this level, the MAOT prediction at $24.146 \mathrm{eV}$ compared fairly well with the DCR calculations [5] equal to $24.138 \mathrm{eV}$. A new measurement or calculation is needed to clarify this uncertain ALS value [4]. Overall, for the entire data quoted in Tables 2-8, comparisons indicate that the MAOT formula reproduces with a very good accuracy the ALS measurements [4] via a simple formalism without using computational codes in contrast with the DCR formalism [5]. Tables 9-11 list resonance energies of the 
Table 1. Resonance energies of the $\left[3 \mathrm{~s}^{2} 3 \mathrm{p}^{3}\left({ }^{2} \mathrm{D}_{5 / 2}^{0}\right)\right] n d$ Rydberg series originating from the $3 \mathrm{~s}^{2} 3 \mathrm{p}^{43} \mathrm{P}_{2}^{\circ}$ ground state of the $\mathrm{Cl}^{+}$ions converging to the $3 \mathrm{~s}^{2} 3 \mathrm{p}^{3}\left({ }^{2} \mathrm{D}_{5 / 2}^{0}\right)$ threshold of $\mathrm{Cl}^{2+}$. The present results from the Modified atomic orbital theory (MAOT) are compared with the Dirac-Coulomb $R$-matrix (DCR) calculations of McLaughlin [5] and with the ALS experimental data of Hernández et al., [4]. The ALS experimental resonance energies are calibrated to $\pm 0.013 \mathrm{eV}$. The energy limits is taken from the NIST tabulations of Ralchenko et al., [19]. $\sigma_{1}\left({ }^{2} \mathrm{D}_{5 / 2}\right)=-0.770 \pm 0.048 ; \sigma_{2}\left({ }^{2} \mathrm{D}_{5 / 2}\right)=15.00$.

\begin{tabular}{|c|c|c|c|c|c|c|}
\hline \multirow{2}{*}{$n$} & MAOT & DCR & ALS & MAOT & DCR & ALS \\
\hline & $E$ & $E$ & $E$ & $\delta$ & $\delta$ & $\delta$ \\
\hline 6 & 24.348 & 24.353 & 24.348 & 0.362 & 0.30 & 0.38 \\
\hline 7 & 24.824 & 24.846 & 24.829 & 0.363 & 0.35 & 0.35 \\
\hline 8 & 25.127 & 25.130 & 25.128 & 0.362 & 0.30 & 0.36 \\
\hline 9 & 25.331 & 25.334 & 25.335 & 0.361 & 0.34 & 0.34 \\
\hline 10 & 25.474 & 25.476 & 25.479 & 0.360 & 0.35 & 0.32 \\
\hline 11 & 25.579 & 25.584 & 25.583 & 0.360 & 0.30 & 0.32 \\
\hline 12 & 25.658 & & & 0.359 & & \\
\hline 13 & 25.719 & & & 0.359 & & \\
\hline 14 & 25.768 & & & 0.358 & & \\
\hline 15 & 25.806 & & & 0.358 & & \\
\hline 16 & 25.838 & & & 0.358 & & \\
\hline 17 & 25.863 & & & 0.358 & & \\
\hline 18 & 25.885 & & & 0.358 & & \\
\hline 19 & 25.903 & & & 0.358 & & \\
\hline 20 & 25.919 & & & 0.358 & & \\
\hline 21 & 25.932 & & & 0.359 & & \\
\hline 22 & 25.944 & & & 0.359 & & \\
\hline 23 & 25.954 & & & 0.359 & & \\
\hline 24 & 25.963 & & & 0.359 & & \\
\hline 25 & 25.970 & & & 0.359 & & \\
\hline 26 & 25.977 & & & 0.360 & & \\
\hline 27 & 25.983 & & & 0.360 & & \\
\hline 28 & 25.989 & & & 0.360 & & \\
\hline 29 & 25.994 & & & 0.361 & & \\
\hline 30 & 25.998 & & & 0.361 & & \\
\hline 31 & 26.002 & & & 0.361 & & \\
\hline 32 & 26.006 & & & 0.361 & & \\
\hline 33 & 26.009 & & & 0.362 & & \\
\hline 34 & 26.012 & & & 0.362 & & \\
\hline 35 & 26.015 & & & 0.362 & & \\
\hline 36 & 26.017 & & & 0.363 & & \\
\hline 37 & 26.019 & & & 0.363 & & \\
\hline 38 & 26.022 & & & 0.363 & & \\
\hline 39 & 26.024 & & & 0.363 & & \\
\hline 40 & 26.025 & & & 0.364 & & \\
\hline$\cdots$ & $\ldots$ & $\ldots$ & $\ldots$ & & $\cdots$ & $\cdots$ \\
\hline$\infty$ & 26.060 & 26.060 & 26.060 & & & \\
\hline
\end{tabular}


Table 2. Resonance energies $(E)$ and quantum defect $(\delta)$ of the $\left[3 \mathrm{~s}^{2} 3 \mathrm{p}^{3}\left({ }^{2} \mathrm{D}_{5 / 2}^{0}\right)\right] n d$ Rydberg series originating from the $3 \mathrm{~s}^{2} 3 \mathrm{p}^{43} \mathrm{P}_{1}^{0}$ metastable state of the $\mathrm{Cl}^{+}$ions converging to the $3 \mathrm{~s}^{2} 3 \mathrm{p}^{3}\left({ }^{2} \mathrm{D}_{5 / 2}^{0}\right)$ threshold of $\mathrm{Cl}^{2+}$. The present results from the Modified atomic orbital theory (MAOT) are compared with the Dirac-Coulomb $R$-matrix (DCR) calculations of McLaughlin [5] and with the ALS experimental data of Hernández et al., [4]. The ALS experimental resonance energies are calibrated to $\pm 0.013 \mathrm{eV}$. The energy limits is taken from the NIST tabulations of Ralchenko et al., [19]. $\sigma_{1}\left({ }^{2} \mathrm{D}_{5 / 2}\right)=-0.781 \pm 0.048$; $\sigma_{2}\left({ }^{2} \mathrm{D}_{5 / 2}\right)=15.00$.

\begin{tabular}{|c|c|c|c|c|c|c|}
\hline \multirow{2}{*}{$n$} & MAOT & DCR & ALS & MAOT & DCR & ALS \\
\hline & $E$ & $E$ & $E$ & $\delta$ & $\delta$ & $\delta$ \\
\hline 6 & 24.259 & 24.264 & 24.259 & 0.367 & 0.36 & 0.37 \\
\hline 7 & 24.737 & 24.762 & 24.750 & 0.368 & 0.30 & 0.33 \\
\hline 8 & 25.040 & 25.039 & 25.036 & 0.367 & 0.37 & 0.38 \\
\hline 9 & 25.244 & 25.237 & 25.238 & 0.366 & 0.40 & 0.40 \\
\hline 10 & 25.388 & 25.385 & 25.384 & 0.365 & 0.40 & 0.42 \\
\hline 11 & 25.493 & 25.493 & (25.492) & 0.365 & 0.36 & $(0.37)$ \\
\hline 12 & 25.572 & & & 0.364 & & \\
\hline 13 & 25.633 & & & 0.363 & & \\
\hline 14 & 25.681 & & & 0.363 & & \\
\hline 15 & 25.720 & & & 0.363 & & \\
\hline 16 & 25.751 & & & 0.363 & & \\
\hline 17 & 25.777 & & & 0.363 & & \\
\hline 18 & 25.799 & & & 0.363 & & \\
\hline 19 & 25.817 & & & 0.363 & & \\
\hline 20 & 25.833 & & & 0.363 & & \\
\hline 21 & 25.846 & & & 0.363 & & \\
\hline 22 & 25.858 & & & 0.364 & & \\
\hline 23 & 25.868 & & & 0.364 & & \\
\hline 24 & 25.877 & & & 0.364 & & \\
\hline 25 & 25.884 & & & 0.364 & & \\
\hline 26 & 25.891 & & & 0.365 & & \\
\hline 27 & 25.897 & & & 0.365 & & \\
\hline 28 & 25.903 & & & 0.365 & & \\
\hline 29 & 25.908 & & & 0.365 & & \\
\hline 30 & 25.912 & & & 0.366 & & \\
\hline 31 & 25.916 & & & 0.366 & & \\
\hline 32 & 25.920 & & & 0.366 & & \\
\hline 33 & 25.923 & & & 0.367 & & \\
\hline 34 & 25.926 & & & 0.367 & & \\
\hline 35 & 25.929 & & & 0.367 & & \\
\hline 36 & 25.931 & & & 0.367 & & \\
\hline 37 & 25.933 & & & 0.368 & & \\
\hline 38 & 25.936 & & & 0.368 & & \\
\hline 39 & 25.938 & & & 0.368 & & \\
\hline 40 & 25.939 & & & 0.369 & & \\
\hline$\ldots$ & $\ldots$ & $\ldots$ & $\ldots$ & & $\ldots$ & $\ldots$ \\
\hline$\infty$ & 25.974 & 25.974 & 25.974 & & & \\
\hline
\end{tabular}


Table 3. Resonance energies $(E)$ and quantum defect $(\delta)$ of the $\left[3 \mathrm{~s}^{2} 3 \mathrm{p}^{3}\left({ }^{2} \mathrm{D}_{5 / 2}^{\circ}\right)\right] n d$ Rydberg series originating from the $3 \mathrm{~s}^{2} 3 \mathrm{p}^{43} \mathrm{P}_{0}^{0}$ metastable state of the $\mathrm{Cl}^{+}$ions converging to the $3 \mathrm{~s}^{2} 3 \mathrm{p}^{3}\left({ }^{2} \mathrm{D}_{5 / 2}^{0}\right)$ threshold of $\mathrm{Cl}^{2+}$. The present results from the Modified atomic orbital theory (MAOT) are compared with the Dirac-Coulomb $R$-matrix (DCR) calculations of McLaughlin [5] and with the ALS experimental data of Hernández et al., [4]. The ALS experimental resonance energies are calibrated to $\pm 0.013 \mathrm{eV}$. The energy limits is taken from the NIST tabulations of Ralchenko et al., [19]. $\sigma_{1}\left({ }^{2} \mathrm{D}_{5 / 2}\right)=-0.789 \pm 0.048$; $\sigma_{2}\left({ }^{2} \mathrm{D}_{5 / 2}\right)=15.00$.

\begin{tabular}{|c|c|c|c|c|c|c|}
\hline \multirow{2}{*}{$n$} & MAOT & DCR & ALS & MAOT & DCR & ALS \\
\hline & $E$ & $E$ & $E$ & $\delta$ & $\delta$ & $\delta$ \\
\hline 6 & 24.219 & 24.223 & 24.219 & 0.370 & 0.36 & 0.37 \\
\hline 7 & 24.697 & 24.733 & & 0.372 & 0.27 & \\
\hline 8 & 25.001 & 24.999 & $(25.000)$ & 0.371 & 0.38 & $(0.38)$ \\
\hline 9 & 25.205 & 25.198 & & 0.370 & 0.40 & \\
\hline 10 & 25.349 & 25.345 & & 0.369 & 0.40 & \\
\hline 11 & 25.455 & & & 0.368 & & \\
\hline 12 & 25.534 & & & 0.367 & & \\
\hline 13 & 25.595 & & & 0.367 & & \\
\hline 14 & 25.643 & & & 0.367 & & \\
\hline 15 & 25.682 & & & 0.366 & & \\
\hline 16 & 25.713 & & & 0.366 & & \\
\hline 17 & 25.739 & & & 0.366 & & \\
\hline 18 & 25.761 & & & 0.366 & & \\
\hline 19 & 25.779 & & & 0.367 & & \\
\hline 20 & 25.795 & & & 0.367 & & \\
\hline 21 & 25.808 & & & 0.367 & & \\
\hline 22 & 25.820 & & & 0.367 & & \\
\hline 23 & 25.830 & & & 0.367 & & \\
\hline 24 & 25.839 & & & 0.368 & & \\
\hline 25 & 25.846 & & & 0.368 & & \\
\hline 26 & 25.853 & & & 0.368 & & \\
\hline 27 & 25.859 & & & 0.368 & & \\
\hline 28 & 25.865 & & & 0.369 & & \\
\hline 29 & 25.870 & & & 0.369 & & \\
\hline 30 & 25.874 & & & 0.369 & & \\
\hline 31 & 25.878 & & & 0.370 & & \\
\hline 32 & 25.882 & & & 0.370 & & \\
\hline 33 & 25.885 & & & 0.370 & & \\
\hline 34 & 25.888 & & & 0.371 & & \\
\hline 35 & 25.891 & & & 0.371 & & \\
\hline 36 & 25.893 & & & 0.371 & & \\
\hline 37 & 25.895 & & & 0.371 & & \\
\hline 38 & 25.898 & & & 0.372 & & \\
\hline 39 & 25.900 & & & 0.372 & & \\
\hline 40 & 25.901 & & & 0.372 & & \\
\hline$\cdots$ & $\cdots$ & $\cdots$ & $\cdots$ & & $\ldots$ & $\ldots$ \\
\hline$\infty$ & 25.936 & 25.936 & 25.936 & & & \\
\hline
\end{tabular}


Table 4. Resonance energies $(E)$ and quantum defect $(\delta)$ of the $\left[3 \mathrm{~s}^{2} 3 \mathrm{p}^{3}\left({ }^{2} \mathrm{P}_{3 / 2}^{0}\right)\right] n d$ Rydberg series originating from the $3 \mathrm{~s}^{2} 3 \mathrm{p}^{43} \mathrm{P}_{2}^{\circ}$ ground state of the $\mathrm{Cl}^{+}$ions converging to the $3 \mathrm{~s}^{2} 3 \mathrm{p}^{3}\left({ }^{2} \mathrm{P}_{3 / 2}^{\circ}\right)$ threshold of $\mathrm{Cl}^{2+}$. The present results from the Modified atomic orbital theory (MAOT) are compared with the Dirac-Coulomb $R$-matrix (DCR) calculations of McLaughlin [5] and with the ALS experimental data of Hernández et al., [4]. The ALS experimental resonance energies are calibrated to $\pm 0.013 \mathrm{eV}$. The energy limits is taken from the NIST tabulations of Ralchenko et al., [19]. $\sigma_{1}\left({ }^{2} \mathrm{P}_{3 / 2}\right)=-1.006 \pm 0.077 ; \sigma_{2}\left({ }^{2} \mathrm{P}_{3 / 2}\right)=$ 15.00 .

\begin{tabular}{|c|c|c|c|c|c|c|}
\hline \multirow{2}{*}{$n$} & MAOT & DCR & ALS & MAOT & DCR & ALS \\
\hline & E & E & $E$ & $\delta$ & $\delta$ & $\delta$ \\
\hline 6 & 25.749 & 25.755 & $(25.745)$ & 0.459 & 0.46 & $(0.47)$ \\
\hline 7 & 26.246 & 26.253 & 26.246 & 0.469 & 0.45 & 0.47 \\
\hline 8 & 26.562 & 26.576 & 26.564 & 0.471 & 0.45 & 0.46 \\
\hline 9 & 26.774 & 26.780 & 26.778 & 0.471 & 0.44 & 0.45 \\
\hline 10 & 26.923 & 26.927 & 26.928 & 0.470 & 0.44 & 0.43 \\
\hline 11 & 27.031 & 27.035 & 27.031 & 0.469 & 0.43 & 0.47 \\
\hline 12 & 27.113 & 27.115 & $(27.114)$ & 0.468 & 0.44 & $(0.45)$ \\
\hline 13 & 27.176 & 27.178 & 27.175 & 0.467 & 0.42 & 0.48 \\
\hline 14 & 27.225 & & & 0.467 & & \\
\hline 15 & 27.264 & & & 0.466 & & \\
\hline 16 & 27.296 & & & 0.466 & & \\
\hline 17 & 27.323 & & & 0.466 & & \\
\hline 18 & 27.345 & & & 0.466 & & \\
\hline 19 & 27.364 & & & 0.466 & & \\
\hline 20 & 27.379 & & & 0.466 & & \\
\hline 21 & 27.393 & & & 0.466 & & \\
\hline 22 & 27.405 & & & 0.466 & & \\
\hline 23 & 27.415 & & & 0.466 & & \\
\hline 24 & 27.424 & & & 0.467 & & \\
\hline 25 & 27.432 & & & 0.467 & & \\
\hline 26 & 27.439 & & & 0.467 & & \\
\hline 27 & 27.445 & & & 0.467 & & \\
\hline 28 & 27.450 & & & 0.468 & & \\
\hline 29 & 27.455 & & & 0.468 & & \\
\hline 30 & 27.460 & & & 0.468 & & \\
\hline 31 & 27.464 & & & 0.469 & & \\
\hline 32 & 27.467 & & & 0.469 & & \\
\hline 33 & 27.471 & & & 0.470 & & \\
\hline 34 & 27.474 & & & 0.470 & & \\
\hline 35 & 27.476 & & & 0.470 & & \\
\hline 36 & 27.479 & & & 0.471 & & \\
\hline 37 & 27.481 & & & 0.471 & & \\
\hline 38 & 27.483 & & & 0.471 & & \\
\hline 39 & 27.485 & & & 0.472 & & \\
\hline 40 & 27.487 & & & 0.472 & & \\
\hline$\cdots$ & $\cdots$ & $\cdots$ & $\cdots$ & & $\cdots$ & $\cdots$ \\
\hline$\infty$ & 27.522 & 27.522 & 27.522 & & & \\
\hline
\end{tabular}


Table 5. Resonance energies $(E, \mathrm{eV})$, quantum defect $(\delta)$ of the $\left[3 \mathrm{~s}^{2} 3 \mathrm{p}^{3}\left({ }^{2} \mathrm{P}_{3 / 2}^{\circ}\right)\right] n d$ Rydberg series originating from the $3 \mathrm{~s}^{2} 3 \mathrm{p}^{43} \mathrm{P}_{1}^{0}$ metastable state of the $\mathrm{Cl}^{+}$ions converging to the $3 \mathrm{~s}^{2} 3 \mathrm{p}^{3}\left({ }^{2} \mathrm{P}_{3 / 2}^{\circ}\right)$ threshold of $\mathrm{Cl}^{2+}$. The present results from the Modified atomic orbital theory (MAOT) are compared with the Dirac-Coulomb $R$-matrix (DCR) calculations of McLaughlin [5] and with the ALS experimental data of Hernández et al., [4]. The ALS experimental resonance energies are calibrated to $\pm 0.013 \mathrm{eV}$. The energy limits is taken from the NIST tabulations of Ralchenko et al., [19]. $\sigma_{1}\left({ }^{2} \mathrm{P}_{3 / 2}\right)=-1.018 \pm 0.076$; $\sigma_{2}\left({ }^{2} \mathrm{P}_{3 / 2}\right)=15.00$.

\begin{tabular}{|c|c|c|c|c|c|c|}
\hline \multirow{2}{*}{$n$} & MAOT & DCR & ALS & MAOT & DCR & ALS \\
\hline & $E$ & E & E & $\delta$ & $\delta$ & $\delta$ \\
\hline 6 & 25.659 & 25.668 & $(25.660)$ & 0.464 & 0.45 & $(0.45)$ \\
\hline 7 & 26.157 & 26.162 & 26.157 & 0.474 & 0.46 & 0.47 \\
\hline 8 & 26.473 & 26.480 & 26.475 & 0.477 & 0.45 & 0.47 \\
\hline 9 & 26.686 & 26.695 & 26.687 & 0.476 & 0.43 & 0.46 \\
\hline 10 & 26.835 & 26.842 & 26.833 & 0.475 & 0.42 & 0.49 \\
\hline 11 & 26.944 & & & 0.474 & & \\
\hline 12 & 27.025 & & & 0.473 & & \\
\hline 13 & 27.088 & & & 0.472 & & \\
\hline 14 & 27.138 & & & 0.472 & & \\
\hline 15 & 27.177 & & & 0.471 & & \\
\hline 16 & 27.209 & & & 0.471 & & \\
\hline 17 & 27.236 & & & 0.471 & & \\
\hline 18 & 27.258 & & & 0.471 & & \\
\hline 19 & 27.276 & & & 0.471 & & \\
\hline 20 & 27.292 & & & 0.471 & & \\
\hline 21 & 27.306 & & & 0.471 & & \\
\hline 22 & 27.318 & & & 0.471 & & \\
\hline 23 & 27.328 & & & 0.471 & & \\
\hline 24 & 27.337 & & & 0.472 & & \\
\hline 25 & 27.345 & & & 0.472 & & \\
\hline 26 & 27.351 & & & 0.472 & & \\
\hline 27 & 27.358 & & & 0.473 & & \\
\hline 28 & 27.363 & & & 0.473 & & \\
\hline 29 & 27.368 & & & 0.473 & & \\
\hline 30 & 27.373 & & & 0.474 & & \\
\hline 31 & 27.377 & & & 0.474 & & \\
\hline 32 & 27.380 & & & 0.475 & & \\
\hline 33 & 27.384 & & & 0.475 & & \\
\hline 34 & 27.387 & & & 0.475 & & \\
\hline 35 & 27.389 & & & 0.476 & & \\
\hline 36 & 27.392 & & & 0.476 & & \\
\hline 37 & 27.394 & & & 0.476 & & \\
\hline 38 & 27.396 & & & 0.477 & & \\
\hline 39 & 27.398 & & & 0.477 & & \\
\hline 40 & 27.400 & & & 0.477 & & \\
\hline$\cdots$ & $\cdots$ & $\ldots$ & $\cdots$ & & $\cdots$ & $\cdots$ \\
\hline$\infty$ & 27.435 & 27.435 & 27.435 & & & \\
\hline
\end{tabular}


Table 6. Resonance energies $(E)$ and quantum defect $(\delta)$ of the $\left[3 \mathrm{~s}^{2} 3 \mathrm{p}^{3}\left({ }^{2} \mathrm{P}_{3 / 2}^{\circ}\right)\right] n d$ Rydberg series originating from the $3 \mathrm{~s}^{2} 3 \mathrm{p}^{43} \mathrm{P}_{0}^{0}$ metastable state of the $\mathrm{Cl}^{+}$ions converging to the $3 \mathrm{~s}^{2} 3 \mathrm{p}^{3}\left({ }^{2} \mathrm{P}_{3 / 2}^{\circ}\right)$ threshold of $\mathrm{Cl}^{2+}$. The present results from the Modified atomic orbital theory (MAOT) are compared with the Dirac-Coulomb $R$-matrix (DCR) calculations of McLaughlin [5] and with the ALS experimental data of Hernández et al., [4]. The ALS experimental resonance energies are calibrated to $\pm 0.013 \mathrm{eV}$. The energy limits is taken from the NIST tabulations of Ralchenko et al., [19]. $\sigma_{1}\left({ }^{2} \mathrm{P}_{3 / 2}\right)=-1.088 \pm 0.076$; $\sigma_{2}\left({ }^{2} \mathrm{P}_{32}\right)=15.00$.

\begin{tabular}{|c|c|c|c|c|c|c|}
\hline \multirow{2}{*}{$n$} & MAOT & DCR & ALS & MAOT & DCR & ALS \\
\hline & $E$ & $E$ & $E$ & $\delta$ & $\delta$ & $\delta$ \\
\hline 6 & 25.603 & 25.635 & & 0.493 & 0.44 & \\
\hline 7 & 26.108 & 26.117 & 26.108 & 0.505 & 0.48 & 0.51 \\
\hline 8 & 26.429 & 26.434 & 26.430 & 0.507 & 0.49 & 0.50 \\
\hline 9 & 26.644 & 26.650 & 26.648 & 0.507 & 0.47 & 0.49 \\
\hline 10 & 26.794 & 26.797 & & 0.506 & 0.48 & \\
\hline 11 & 26.904 & & & 0.504 & & \\
\hline 12 & 26.986 & & & 0.503 & & \\
\hline 13 & 27.050 & & & 0.502 & & \\
\hline 14 & 27.099 & & & 0.502 & & \\
\hline 15 & 27.139 & & & 0.501 & & \\
\hline 16 & 27.171 & & & 0.501 & & \\
\hline 17 & 27.198 & & & 0.501 & & \\
\hline 18 & 27.220 & & & 0.501 & & \\
\hline 19 & 27.239 & & & 0.501 & & \\
\hline 20 & 27.255 & & & 0.501 & & \\
\hline 21 & 27.268 & & & 0.501 & & \\
\hline 22 & 27.280 & & & 0.501 & & \\
\hline 23 & 27.290 & & & 0.502 & & \\
\hline 24 & 27.299 & & & 0.502 & & \\
\hline 25 & 27.307 & & & 0.502 & & \\
\hline 26 & 27.314 & & & 0.503 & & \\
\hline 27 & 27.320 & & & 0.503 & & \\
\hline 28 & 27.326 & & & 0.504 & & \\
\hline 29 & 27.331 & & & 0.504 & & \\
\hline 30 & 27.335 & & & 0.504 & & \\
\hline 31 & 27.339 & & & 0.505 & & \\
\hline 32 & 27.343 & & & 0.505 & & \\
\hline 33 & 27.346 & & & 0.506 & & \\
\hline 34 & 27.349 & & & 0.506 & & \\
\hline 35 & 27.352 & & & 0.507 & & \\
\hline 36 & 27.355 & & & 0.507 & & \\
\hline 37 & 27.357 & & & 0.507 & & \\
\hline 38 & 27.359 & & & 0.508 & & \\
\hline 39 & 27.361 & & & 0.508 & & \\
\hline 40 & 27.363 & & & 0.509 & & \\
\hline$\cdots$ & $\ldots$ & $\cdots$ & $\ldots$ & & $\ldots$ & $\ldots$ \\
\hline$\infty$ & 27.398 & 27.398 & 27.398 & & & \\
\hline
\end{tabular}


Table 7. Resonance energies $(E, \mathrm{eV})$, quantum defect $(\delta)$ of the $\left[3 \mathrm{~s}^{2} 3 \mathrm{p}^{3}\left({ }^{2} \mathrm{P}_{1 / 2}^{\circ}\right)\right] n d$ Rydberg series originating from the $3 \mathrm{~s}^{2} 3 \mathrm{p}^{4} \mathrm{~S}_{0}^{\circ}$ metastable state of the $\mathrm{Cl}^{+}$ions converging to the $3 \mathrm{~s}^{2} 3 \mathrm{p}^{3}\left({ }^{2} \mathrm{P}_{1 / 2}^{\circ}\right)$ threshold of $\mathrm{Cl}^{2+}$. The present results from the Modified atomic orbital theory (MAOT) are compared with the Dirac-Coulomb $R$-matrix (DCR) calculations of McLaughlin [5] and with the ALS experimental data of Hernández et al., [4]. The ALS experimental resonance energies are calibrated to $\pm 0.013 \mathrm{eV}$. The energy limits is taken from the NIST tabulations of Ralchenko et al., [19]. $\sigma_{1}\left({ }^{2} \mathrm{P}_{1 / 2}\right)=-0.262 \pm 0.015 ; \sigma_{2}\left({ }^{2} \mathrm{P}_{1 / 2}\right)=$ 15.00 .

\begin{tabular}{|c|c|c|c|c|c|c|}
\hline \multirow{2}{*}{$n$} & MAOT & DCR & ALS & MAOT & DCR & ALS \\
\hline & $E$ & $E$ & $E$ & $\delta$ & $\delta$ & $\delta$ \\
\hline 4 & 20.426 & 20.420 & 20.426 & 0.127 & 0.13 & 0.13 \\
\hline 5 & 21.762 & 21.734 & 21.742 & 0.127 & 0.16 & 0.15 \\
\hline 6 & 22.477 & 22.459 & 22.463 & 0.126 & 0.16 & 0.15 \\
\hline 7 & 22.902 & 22.890 & 22.900 & 0.126 & 0.16 & 0.13 \\
\hline 8 & 23.176 & 23.170 & 23.178 & 0.125 & 0.15 & 0.12 \\
\hline 9 & 23.363 & 23.361 & & 0.125 & 0.14 & \\
\hline 10 & 23.496 & & & 0.125 & & \\
\hline 11 & 23.594 & & & 0.124 & & \\
\hline 12 & 23.668 & & & 0.124 & & \\
\hline 13 & 23.726 & & & 0.124 & & \\
\hline 14 & 23.771 & & & 0.124 & & \\
\hline 15 & 23.808 & & & 0.124 & & \\
\hline 16 & 23.838 & & & 0.124 & & \\
\hline 17 & 23.863 & & & 0.124 & & \\
\hline 18 & 23.884 & & & 0.124 & & \\
\hline 19 & 23.901 & & & 0.124 & & \\
\hline 20 & 23.916 & & & 0.124 & & \\
\hline 21 & 23.929 & & & 0.124 & & \\
\hline 22 & 23.940 & & & 0.124 & & \\
\hline 23 & 23.950 & & & 0.124 & & \\
\hline 24 & 23.959 & & & 0.124 & & \\
\hline 25 & 23.966 & & & 0.124 & & \\
\hline 26 & 23.973 & & & 0.125 & & \\
\hline 27 & 23.979 & & & 0.125 & & \\
\hline 28 & 23.984 & & & 0.125 & & \\
\hline 29 & 23.989 & & & 0.125 & & \\
\hline 30 & 23.993 & & & 0.125 & & \\
\hline 31 & 23.997 & & & 0.125 & & \\
\hline 32 & 24.000 & & & 0.125 & & \\
\hline 33 & 24.004 & & & 0.125 & & \\
\hline 34 & 24.007 & & & 0.125 & & \\
\hline 35 & 24.009 & & & 0.125 & & \\
\hline 36 & 24.012 & & & 0.125 & & \\
\hline 37 & 24.014 & & & 0.125 & & \\
\hline$\cdots$ & $\cdots$ & $\cdots$ & $\ldots$ & & $\ldots$ & $\cdots$ \\
\hline$\infty$ & 24.054 & 24.054 & 24.054 & & & \\
\hline
\end{tabular}


Table 8. Resonance energies $(E)$ and quantum defect $(\delta)$ of the $\left[3 \mathrm{~s}^{2} 3 \mathrm{p}^{3}\left({ }^{2} \mathrm{D}_{5 / 2}^{0}\right)\right] n$ d series originating from the $3 \mathrm{~s}^{2} 3 \mathrm{p}^{41} \mathrm{D}_{2}$ metastable state of the $\mathrm{Cl}^{+}$ions converging to the $3 \mathrm{~s}^{2} 3 \mathrm{p}^{3}\left({ }^{2} \mathrm{D}_{5 / 2}^{0}\right)$ threshold of $\mathrm{Cl}^{2+}$. The present results from the Modified atomic orbital theory (MAOT) are compared with the Dirac-Coulomb $R$-matrix (DCR) calculations of McLaughlin [5] and with the ALS experimental data of Hernández et al., [4]. The ALS experimental resonance energies are calibrated to $\pm 0.013 \mathrm{eV}$. The energy limits is taken from the NIST tabulations of Ralchenko et al., [19]. $\sigma_{1}\left({ }^{2} \mathrm{D}_{5 / 2}\right)=-0.483 \pm 0.050 ; \sigma_{2}\left({ }^{2} \mathrm{D}_{5 / 2}\right)=$ 15.00 .

\begin{tabular}{|c|c|c|c|c|c|c|}
\hline \multirow{2}{*}{$n$} & MAOT & DCR & ALS & MAOT & DCR & ALS \\
\hline & E & E & $E$ & $\delta$ & $\delta$ & $\delta$ \\
\hline 6 & 22.979 & 22.969 & 22.979 & 0.232 & 0.25 & 0.27 \\
\hline 7 & 23.427 & 23.392 & & 0.233 & 0.32 & \\
\hline 8 & 23.713 & 23.719 & 23.718 & 0.232 & 0.21 & 0.21 \\
\hline 9 & 23.907 & 23.907 & 23.907 & 0.232 & 0.23 & 0.23 \\
\hline 10 & 24.045 & 24.039 & 24.040 & 0.231 & 0.28 & 0.27 \\
\hline 11 & 24.146 & 24.138 & $(24.152)$ & 0.231 & 0.32 & $(0.27)$ \\
\hline 12 & 24.222 & & & 0.230 & & \\
\hline 13 & 24.281 & & & 0.230 & & \\
\hline 14 & 24.328 & & & 0.230 & & \\
\hline 15 & 24.366 & & & 0.230 & & \\
\hline 16 & 24.396 & & & 0.230 & & \\
\hline 17 & 24.421 & & & 0.229 & & \\
\hline 18 & 24.443 & & & 0.229 & & \\
\hline 19 & 24.461 & & & 0.229 & & \\
\hline 20 & 24.476 & & & 0.229 & & \\
\hline 21 & 24.489 & & & 0.229 & & \\
\hline 22 & 24.500 & & & 0.229 & & \\
\hline 23 & 24.510 & & & 0.229 & & \\
\hline 24 & 24.519 & & & 0.230 & & \\
\hline 25 & 24.526 & & & 0.230 & & \\
\hline 26 & 24.533 & & & 0.230 & & \\
\hline 27 & 24.539 & & & 0.230 & & \\
\hline 28 & 24.544 & & & 0.230 & & \\
\hline 29 & 24.549 & & & 0.230 & & \\
\hline 30 & 24.554 & & & 0.230 & & \\
\hline 31 & 24.558 & & & 0.230 & & \\
\hline 32 & 24.561 & & & 0.230 & & \\
\hline 33 & 24.564 & & & 0.230 & & \\
\hline 34 & 24.567 & & & 0.231 & & \\
\hline 35 & 24.570 & & & 0.231 & & \\
\hline 36 & 24.572 & & & 0.231 & & \\
\hline 37 & 24.575 & & & 0.231 & & \\
\hline 38 & 24.577 & & & 0.231 & & \\
\hline 39 & 24.579 & & & 0.231 & & \\
\hline 40 & 24.581 & & & 0.231 & & \\
\hline$\cdots$ & $\ldots$ & $\ldots$ & $\ldots$ & & $\ldots$ & $\ldots$ \\
\hline$\infty$ & 24.615 & 24.615 & 24.615 & & & \\
\hline
\end{tabular}


Table 9. Resonance energies $(E)$ and quantum defect $(\delta)$ of the $3 s^{2} 3 \mathrm{p}^{4}\left({ }^{1} \mathrm{D}_{2}\right) n s(j=1 / 2$ series originating from the $3 \mathrm{~s}^{2} 3 \mathrm{p}^{52} \mathrm{P}_{1 / 2}^{0}$ metastable state of the $\mathrm{Ar}^{+}$ions converging to the ${ }^{1} \mathrm{D}_{2}$ threshold of $\mathrm{Ar}^{2+}$. The present results from the Modified atomic orbital theory (MAOT) are compared with the Screening constant by unit nuclear charge (SCUNC) results of Sakho [11] are compared with the QB data of Covington et al., [9]. The energy limits $\mathrm{eV}$ ) is taken from the NIST tabulations of Ralchenko et al., [17]. $\sigma_{1}\left({ }^{1} \mathrm{D}_{2}\right)=-4.227 \pm$ $0.224 ; \sigma_{2}\left({ }^{1} \mathrm{D}_{2}\right)=16.00$.

\begin{tabular}{|c|c|c|c|c|c|c|}
\hline \multirow{2}{*}{$n$} & MAOT & SCUNC & QB & MAOT & SCUNC & QB \\
\hline & $E$ & $E$ & $E$ & $\delta$ & $\delta$ & $\delta$ \\
\hline 8 & 27.830 & 27.830 & 27.830 & 1.672 & 1.673 & 1.673 \\
\hline 9 & 28.169 & 28.173 & 28.173 & 1.695 & 1.682 & 1.682 \\
\hline 10 & 28.400 & 28.401 & 28.401 & 1.696 & 1.689 & 1.688 \\
\hline 11 & 28.561 & 28.561 & 28.561 & 1.689 & 1.694 & 1.692 \\
\hline 12 & 28.678 & 28.676 & 28.677 & 1.679 & 1.697 & 1.695 \\
\hline 13 & 28.765 & 28.763 & 28.763 & 1.670 & 1.699 & 1.698 \\
\hline 14 & 28.831 & 28.829 & 28.829 & 1.662 & 1.700 & 1.699 \\
\hline 15 & 28.883 & 28.881 & 28.881 & 1.656 & 1.701 & 1.701 \\
\hline 16 & 28.925 & 28.923 & 28.923 & 1.652 & 1.701 & 1.702 \\
\hline 17 & 28.958 & 28.956 & & 1.649 & 1.700 & \\
\hline 18 & 28.985 & 28.984 & & 1.647 & 1.700 & \\
\hline 19 & 29.008 & 29.007 & & 1.647 & 1.698 & \\
\hline 20 & 29.027 & 29.027 & & 1.648 & 1.697 & \\
\hline 21 & 29.044 & 29.043 & & 1.649 & 1.695 & \\
\hline 22 & 29.058 & 29.057 & & 1.652 & 1.693 & \\
\hline 23 & 29.070 & 29.069 & & 1.655 & 1.691 & \\
\hline 24 & 29.080 & 29.080 & & 1.658 & 1.688 & \\
\hline 25 & 29.089 & 29.089 & & 1.662 & 1.686 & \\
\hline 26 & 29.097 & 29.097 & & 1.666 & 1.683 & \\
\hline 27 & 29.104 & 29.104 & & 1.670 & 1.680 & \\
\hline 28 & 29.110 & 29.110 & & 1.675 & 1.677 & \\
\hline 29 & 29.116 & 29.116 & & 1.679 & 1.674 & \\
\hline 30 & 29.121 & 29.121 & & 1.684 & 1.671 & \\
\hline 31 & 29.126 & & & 1.689 & & \\
\hline 32 & 29.130 & & & 1.694 & & \\
\hline 33 & 29.133 & & & 1.699 & & \\
\hline 34 & 29.137 & & & 1.704 & & \\
\hline 35 & 29.140 & & & 1.708 & & \\
\hline 36 & 29.143 & & & 1.713 & & \\
\hline 37 & 29.145 & & & 1.718 & & \\
\hline 38 & 29.148 & & & 1.723 & & \\
\hline 39 & 29.150 & & & 1.727 & & \\
\hline 40 & 29.152 & & & 1.732 & & \\
\hline$\ldots$ & $\ldots$ & $\ldots$ & $\ldots$ & & $\ldots$ & $\ldots$ \\
\hline$\infty$ & 29.189 & 29.189 & 29.189 & & & \\
\hline
\end{tabular}


Table 10. Resonance energies $(E)$ and quantum defect $(\delta)$ of the $3 \mathrm{~s}^{2} 3 \mathrm{p}^{4}\left({ }^{1} \mathrm{D}_{2}\right) n \mathrm{~d}(j=1 / 2$ series originating from the $3 \mathrm{~s}^{2} 3 \mathrm{p}^{52} \mathrm{P}_{1 / 2}^{0}$ metastable state of the $\mathrm{Ar}^{+}$ions converging to the $3 s^{2} 3 \mathrm{p}^{4}\left({ }^{1} \mathrm{D}_{2}\right)$ threshold of $\mathrm{Ar}^{2+}$. The present results from the Modified atomic orbital theory (MAOT) are compared with the Screening constant by unit nuclear charge (SCUNC) results of Sakho [11] are compared with the QB data of Covington et al., [9]. The energy limits $\mathrm{eV})$ is taken from the NIST tabulations of Ralchenko et al., [17]. $\sigma_{1}\left({ }^{1} \mathrm{D}_{2}\right)=-1.159 \pm$ $0.265 ; \sigma_{2}\left({ }^{1} \mathrm{D}_{2}\right)=16.00$.

\begin{tabular}{|c|c|c|c|c|c|c|}
\hline \multirow{2}{*}{$n$} & MAOT & SCUNC & QB & MAOT & SCUNC & QB \\
\hline & $E$ & $E$ & $E$ & $\delta$ & $\delta$ & $\delta$ \\
\hline 8 & 28.211 & 28.211 & 28.211 & 0.540 & 0.540 & 0.540 \\
\hline 9 & 28.428 & 28.426 & 28.426 & 0.543 & 0.555 & 0.554 \\
\hline 10 & 28.581 & 28.578 & 28.576 & 0.543 & 0.563 & 0.574 \\
\hline 11 & 28.691 & 28.689 & 28.691 & 0.542 & 0.568 & 0.546 \\
\hline 12 & 28.775 & 28.772 & 28.773 & 0.541 & 0.571 & 0.562 \\
\hline 13 & 28.838 & 28.837 & 28.837 & 0.540 & 0.573 & 0.568 \\
\hline 14 & 28.889 & 28.887 & 28.887 & 0.539 & 0.573 & 0.572 \\
\hline 15 & 28.929 & 28.928 & 28.928 & 0.538 & 0.573 & 0.574 \\
\hline 16 & 28.961 & 28.960 & & 0.538 & 0.572 & \\
\hline 17 & 28.988 & 28.987 & & 0.537 & 0.571 & \\
\hline 18 & 29.011 & 29.010 & & 0.537 & 0.570 & \\
\hline 19 & 29.029 & 29.029 & & 0.537 & 0.568 & \\
\hline 20 & 29.045 & 29.045 & & 0.537 & 0.567 & \\
\hline 21 & 29.059 & 29.059 & & 0.537 & 0.565 & \\
\hline 22 & 29.071 & 29.071 & & 0.537 & 0.563 & \\
\hline 23 & 29.081 & 29.081 & & 0.537 & 0.560 & \\
\hline 24 & 29.090 & 29.090 & & 0.537 & 0.558 & \\
\hline 25 & 29.098 & 29.098 & & 0.538 & 0.556 & \\
\hline 26 & 29.105 & 29.105 & & 0.538 & 0.553 & \\
\hline 27 & 29.111 & 29.111 & & 0.538 & 0.551 & \\
\hline 28 & 29.117 & 29.117 & & 0.538 & 0.548 & \\
\hline 29 & 29.122 & 29.122 & & 0.539 & 0.546 & \\
\hline 30 & 29.126 & 29.126 & & 0.539 & 0.543 & \\
\hline 31 & 29.130 & & & 0.539 & & \\
\hline 32 & 29.134 & & & 0.540 & & \\
\hline 33 & 29.137 & & & 0.540 & & \\
\hline 34 & 29.140 & & & 0.540 & & \\
\hline 35 & 29.143 & & & 0.541 & & \\
\hline 36 & 29.146 & & & 0.541 & & \\
\hline 37 & 29.148 & & & 0.542 & & \\
\hline 38 & 29.150 & & & 0.542 & & \\
\hline 39 & 29.152 & & & 0.542 & & \\
\hline 40 & 29.154 & & & 0.543 & & \\
\hline$\cdots$ & $\ldots$ & $\ldots$ & $\ldots$ & & $\cdots$ & $\ldots$ \\
\hline$\infty$ & 29.189 & 29.189 & 29.189 & & & \\
\hline
\end{tabular}


Table 11. Resonance energies $(E)$ and quantum defect $(\delta)$ of the $3 \mathrm{~s}^{2} 3 \mathrm{p}^{4}\left({ }^{1} \mathrm{D}_{2}\right)$ nd $(j=3 / 2$ series originating from the $3 \mathrm{~s}^{2} 3 \mathrm{p}^{52} \mathrm{P}_{1 / 2}^{\circ}$ metastable state of the $\mathrm{Ar}^{+}$ions converging to the $3 \mathrm{~s}^{2} 3 \mathrm{p}^{4}\left({ }^{1} \mathrm{D}_{2}\right)$ threshold of $\mathrm{Ar}^{2+}$. The present results from the Modified atomic orbital theory (MAOT) are compared with the Screening constant by unit nuclear charge (SCUNC) results of Sakho [11] are compared with the QB data of Covington et al., [9]. The energy limits $\mathrm{eV})$ is taken from the NIST tabulations of Ralchenko et al., [17]. $\sigma_{1}\left({ }^{1} \mathrm{D}_{2}\right)=-1.159 \pm$ $0.265 ; \sigma_{2}\left({ }^{1} \mathrm{D}_{2}\right)=16.00$.

\begin{tabular}{|c|c|c|c|c|c|c|}
\hline \multirow{2}{*}{$n$} & MAOT & SCUNC & QB & MAOT & SCUNC & QB \\
\hline & $E$ & $E$ & $E$ & $\delta$ & $\delta$ & $\delta$ \\
\hline 8 & 27.821 & 27.821 & 27.821 & 1.693 & 1.693 & 1.692 \\
\hline 9 & 28.163 & 28.171 & 28.171 & 1.717 & 1.688 & 1.691 \\
\hline 10 & 28.396 & 28.401 & 28.401 & 1.718 & 1.689 & 1.690 \\
\hline 11 & 28.558 & 28.561 & 28.561 & 1.710 & 1.692 & 1.689 \\
\hline 12 & 28.676 & 28.677 & 28.677 & 1.700 & 1.693 & 1.688 \\
\hline 13 & 28.763 & 28.763 & 28.764 & 1.691 & 1.695 & 1.688 \\
\hline 14 & 28.830 & 28.830 & 28.830 & 1.683 & 1.695 & 1.688 \\
\hline 15 & 28.882 & 28.882 & 28.882 & 1.676 & 1.695 & 1.687 \\
\hline 16 & 28.924 & 28.923 & 28.923 & 1.672 & 1.694 & 1.687 \\
\hline 17 & 28.957 & 28.957 & & 1.669 & 1.692 & \\
\hline 18 & 28.985 & 28.984 & & 1.667 & 1.691 & \\
\hline 19 & 29.008 & 29.007 & & 1.667 & 1.688 & \\
\hline 20 & 29.027 & 29.027 & & 1.668 & 1.686 & \\
\hline 21 & 29.043 & 29.043 & & 1.669 & 1.683 & \\
\hline 22 & 29.057 & 29.057 & & 1.672 & 1.679 & \\
\hline 23 & 29.069 & 29.069 & & 1.675 & 1.676 & \\
\hline 24 & 29.080 & 29.080 & & 1.678 & 1.672 & \\
\hline 25 & 29.089 & 29.089 & & 1.682 & 1.668 & \\
\hline 26 & 29.097 & 29.097 & & 1.686 & 1.663 & \\
\hline 27 & 29.104 & 29.104 & & 1.691 & 1.659 & \\
\hline 28 & 29.110 & 29.111 & & 1.695 & 1.655 & \\
\hline 29 & 29.116 & 29.116 & & 1.700 & 1.650 & \\
\hline 30 & 29.121 & 29.121 & & 1.705 & 1.645 & \\
\hline 31 & 29.126 & & & 1.710 & & \\
\hline 32 & 29.130 & & & 1.715 & & \\
\hline 33 & 29.133 & & & 1.720 & & \\
\hline 34 & 29.137 & & & 1.725 & & \\
\hline 35 & 29.140 & & & 1.730 & & \\
\hline 36 & 29.143 & & & 1.735 & & \\
\hline 37 & 29.145 & & & 1.740 & & \\
\hline 38 & 29.148 & & & 1.745 & & \\
\hline 39 & 29.150 & & & 1.750 & & \\
\hline 40 & 29.152 & & & 1.754 & & \\
\hline$\ldots$ & $\cdots$ & $\cdots$ & $\ldots$ & & $\ldots$ & $\cdots$ \\
\hline$\infty$ & 29.189 & 29.189 & 29.189 & & & \\
\hline
\end{tabular}


$3 \mathrm{~s}^{2} 3 \mathrm{p}^{4}\left({ }^{1} \mathrm{D}_{2}\right) n \mathrm{~s}, n d(j=1 / 2)$ and of the $3 \mathrm{~s}^{2} 3 \mathrm{p}^{4}\left({ }^{1} \mathrm{D}_{2}\right) n d(j=3 / 2)$ series originating from the $3 \mathrm{~s}^{2} 3 \mathrm{p}^{5} \mathrm{P}_{1 / 2}^{\circ}$ metastable state of the Ar II ions. Comparison indicate an excellent agreements between the present results from the Modified atomic orbital theory (MAOT) and both the Screening constant by unit nuclear charge (SCUNC) results [11] and the multichannel $R$-matrix QB technique which defines matrices $Q$ and $B$ in terms of asymptotic solutions [9]. These very good agreements are also observed comparing the resonance energies $3 s^{2} 3 \mathrm{p}^{4}\left({ }^{1} \mathrm{D}_{2}\right) n \mathrm{~s}$, nd $(j=1 / 2)$ series originating from the $3 \mathrm{~s}^{2} 3 \mathrm{p}^{5} \mathrm{P}_{3 / 2}^{\circ}$ ground state of the Ar II ions. For both Tables 9-12, the QB data are limited to $n=16$ and the SCUNC values to $n=30$. High lying MAOT data are tabulated up to $n=40$ with a constant quantum defect along each series. Table 13 and Table 14 quote respectively resonance energies of the $3 \mathrm{~s}^{2} 3 \mathrm{p}^{4}\left({ }^{1} \mathrm{D}_{2}\right) n \mathrm{~d}(j=1 / 2)$ and of the $3 \mathrm{~s}^{2} 3 \mathrm{p}^{4}\left({ }^{1} \mathrm{D}_{2}\right) n \mathrm{~s}(j$ $=3 / 2$ ) series originating from the $3 \mathrm{~s}^{2} 3 \mathrm{p}^{52} \mathrm{P}_{3 / 2}^{0}$ ground state of the Ar II ions. Here again, the agreements between the present MAOT results and both SCUNC of Sakho [11] and QB results of Covington et al., [9] are very good. Table 15 presents resonance energies of the $3 s^{2} 3 \mathrm{p}^{4}\left({ }^{1} \mathrm{D}_{2}\right) n d(j=3 / 2)$ series originating from the $3 \mathrm{~s}^{2} 3 \mathrm{p}^{52} \mathrm{P}_{3 / 2}^{0}$ ground state of the Ar II ions. In this Table, an uncertain QB data [9] is quoted at $(28.774 \mathrm{eV})$ for the $3 \mathrm{~s}^{2} 3 \mathrm{p}^{4}\left({ }^{1} \mathrm{D}_{2}\right) 10 \mathrm{~d}$ level. For this level, the MOAT prediction is at $28.735 \mathrm{eV}$ to be compared to the SCUNC forecast at $28.734 \mathrm{eV}$. Subsequently, the QB data at $(28.774 \mathrm{eV})$ associated with a quantum defect equal to (0.422) is less precise. For this level, both the MAOT and SCUNC [11] predictions associated with the quantum defects 0.721 and 0.724 are preferable. Therefore, it should be underlined that the SCUNC calculations are more precise than the MOAT calculations. This is due mainly to the fact that, the SCUNC formalism is a development of $1 / Z$ taking implicitly into account more relativistic effects than the MAOT formalism witch is a simple development on $1 / n$. In addition, in the SCUNC formalism, great accuracy are obtained when performing the analytical formula for each atomic system [11]. In the present work, the same Formula (6) is used for both $\mathrm{Cl}$ II, Ar II and $\mathrm{Kr}$ II in contrast with the work of Sakho [11] where the resonance energy expression for the Ar II ions is different to that of the Kr II ions. Table 16 and Table 17 list natural widths of the $\left[3 \mathrm{~s}^{2} 3 \mathrm{p}^{4}\left({ }^{1} \mathrm{D}_{2}\right)\right] n s$, $n \mathrm{~d}(j=1 / 2)$ (Table 16) and of the $\left[3 \mathrm{~s}^{2} 3 \mathrm{p}^{4}\left({ }^{1} \mathrm{D}_{2}\right)\right] n \mathrm{~s}, n \mathrm{~d}(j=3 / 2)$ (Table 17) series originating from the $3 \mathrm{~s}^{2} 3 \mathrm{p}^{52} \mathrm{P}_{1 / 2}^{0}$ metastable state of $\mathrm{Ar}^{+}$ions. It can be seen that the present MAOT data agree well with both the SCUNC results [11] and QB data [9]. It should be mentioned again that the SCUNC calculations are more precise than the MOAT calculations for the reason explained above. Table 18 lists resonance energies and quantum defect of the $\left[4 \mathrm{~s}^{2} 4 \mathrm{p}^{4}\left({ }^{1} \mathrm{D}_{2}\right)\right] n$ d series originating from the $4 \mathrm{~s}^{2} 4 \mathrm{p}^{52} \mathrm{P}_{3 / 2}^{0}$ ground state of the $\mathrm{Kr}$ II ions. Comparisons indicate very good agreements between the present results from the MAOT formalism and both the SCUNC calculations [11] up to $n=30$ and ALS measurements of [10] up to $n=13$. In this iable, two uncertain ALS data are quoted for the $\left[4 \mathrm{~s}^{2} 4 \mathrm{p}^{4}\left({ }^{1} \mathrm{D}_{2}\right)\right] 12 \mathrm{~d}$ and $\left[4 \mathrm{~s}^{2} 4 \mathrm{p}^{4}\left({ }^{1} \mathrm{D}_{2}\right)\right] 13 \mathrm{~d}$ levels respectively at $(25.880 \mathrm{eV})$ and $(25.926 \mathrm{eV})$. The 
Table 12. Resonance energies $(E)$ and quantum defect $(\delta)$ of the $3 \mathrm{~s}^{2} 3 \mathrm{p}^{4}\left({ }^{1} \mathrm{D}_{2}\right) n \mathrm{~s}(j=1 / 2$ series originating from the $3 \mathrm{~s}^{2} 3 \mathrm{p}^{52} \mathrm{P}_{3 / 2}^{\circ}$ ground state of the $\mathrm{Ar}^{+}$ions converging to the $3 s^{2} 3 \mathrm{p}^{4}\left({ }^{1} \mathrm{D}_{2}\right)$ threshold of $\mathrm{Ar}^{2+}$. The present results from the Modified atomic orbital theory (MAOT) are compared with the Screening constant by unit nuclear charge (SCUNC) results of Sakho [11] are compared with the QB data of Covington et al., [9]. The energy limits $\mathrm{eV})$ is taken from the NIST tabulations of Ralchenko et al., [17]. $\sigma_{1}\left({ }^{1} \mathrm{D}_{2}\right)=-4.234 \pm$ $0.224 ; \sigma_{2}\left({ }^{1} \mathrm{D}_{2}\right)=16.00$.

\begin{tabular}{|c|c|c|c|c|c|c|}
\hline \multirow{2}{*}{$n$} & MAOT & SCUNC & QB & MAOT & SCUNC & QB \\
\hline & $E$ & $E$ & $E$ & $\delta$ & $\delta$ & $\delta$ \\
\hline 8 & 28.007 & 28.007 & 28.007 & 1.674 & 1.674 & 1.673 \\
\hline 9 & 28.346 & 28.351 & 28.351 & 1.698 & 1.681 & 1.682 \\
\hline 10 & 28.577 & 28.579 & 28.579 & 1.699 & 1.688 & 1.688 \\
\hline 11 & 28.739 & 28.739 & 28.739 & 1.691 & 1.694 & 1.692 \\
\hline 12 & 28.856 & 28.854 & 28.854 & 1.681 & 1.698 & 1.696 \\
\hline 13 & 28.943 & 28.941 & 28.941 & 1.672 & 1.701 & 1.698 \\
\hline 14 & 29.009 & 29.007 & 29.007 & 1.664 & 1.702 & 1.700 \\
\hline 15 & 29.061 & 29.059 & 29.059 & 1.658 & 1.702 & 1.701 \\
\hline 16 & 29.103 & 29.101 & 29.100 & 1.654 & 1.702 & 1.702 \\
\hline 17 & 29.136 & 29.135 & & 1.651 & 1.700 & \\
\hline 18 & 29.163 & 29.162 & & 1.649 & 1.698 & \\
\hline 19 & 29.186 & 29.185 & & 1.649 & 1.696 & \\
\hline 20 & 29.205 & 29.205 & & 1.650 & 1.693 & \\
\hline 21 & 29.222 & 29.221 & & 1.652 & 1.690 & \\
\hline 22 & 29.236 & 29.235 & & 1.654 & 1.686 & \\
\hline 23 & 29.248 & 29.247 & & 1.657 & 1.682 & \\
\hline 24 & 29.258 & 29.258 & & 1.660 & 1.678 & \\
\hline 25 & 29.267 & 29.267 & & 1.664 & 1.673 & \\
\hline 26 & 29.275 & 29.275 & & 1.668 & 1.668 & \\
\hline 27 & 29.282 & 29.282 & & 1.672 & 1.663 & \\
\hline 28 & 29.288 & 29.289 & & 1.677 & 1.658 & \\
\hline 29 & 29.294 & 29.294 & & 1.682 & 1.653 & \\
\hline 30 & 29.299 & 29.299 & & 1.686 & 1.647 & \\
\hline 31 & 29.304 & & & 1.691 & & \\
\hline 32 & 29.308 & & & 1.696 & & \\
\hline 33 & 29.311 & & & 1.701 & & \\
\hline 34 & 29.315 & & & 1.706 & & \\
\hline 35 & 29.318 & & & 1.711 & & \\
\hline 36 & 29.321 & & & 1.716 & & \\
\hline 37 & 29.323 & & & 1.720 & & \\
\hline 38 & 29.326 & & & 1.725 & & \\
\hline 39 & 29.328 & & & 1.730 & & \\
\hline 40 & 29.330 & & & 1.734 & & \\
\hline$\ldots$ & $\ldots$ & $\cdots$ & $\cdots$ & & $\cdots$ & $\cdots$ \\
\hline$\infty$ & 29.367 & 29.367 & 29.367 & & & \\
\hline
\end{tabular}


Table 13. Resonance energies $(E)$ and quantum defect $(\delta)$ of the $3 \mathrm{~s}^{2} 3 \mathrm{p}^{4}\left({ }^{1} \mathrm{D}_{2}\right) n \mathrm{~d}(j=1 / 2$ series originating from the $3 \mathrm{~s}^{2} 3 \mathrm{p}^{52} \mathrm{P}_{3 / 2}^{\circ}$ ground state of the $\mathrm{Ar}^{+}$ions converging to the $3 \mathrm{~s}^{2} 3 \mathrm{p}^{4}\left({ }^{1} \mathrm{D}_{2}\right)$ threshold of $\mathrm{Ar}^{2+}$. The present results from the Modified atomic orbital theory (MAOT) are compared with the Screening constant by unit nuclear charge (SCUNC) results of Sakho [11] are compared with the QB data of Covington et al., [9]. The energy limits $\mathrm{eV}$ ) is taken from the NIST tabulations of Ralchenko et al., [17]. $\sigma_{1}\left({ }^{1} \mathrm{D}_{2}\right)=-1.159 \pm$ $0.265 ; \sigma_{2}\left({ }^{1} \mathrm{D}_{2}\right)=16.00$.

\begin{tabular}{|c|c|c|c|c|c|c|}
\hline \multirow{2}{*}{$n$} & MAOT & SCUNC & QB & MAOT & SCUNC & QB \\
\hline & $E$ & $E$ & $E$ & $\delta$ & $\delta$ & $\delta$ \\
\hline 8 & 28.389 & 28.389 & 28.389 & 0.540 & 0.540 & 0.540 \\
\hline 9 & 28.606 & 28.603 & 28.603 & 0.543 & 0.560 & 0.554 \\
\hline 10 & 28.759 & 28.755 & 28.754 & 0.543 & 0.567 & 0.574 \\
\hline 11 & 28.869 & 28.867 & 28.869 & 0.542 & 0.572 & 0.546 \\
\hline 12 & 28.953 & 28.950 & 28.951 & 0.541 & 0.575 & 0.562 \\
\hline 13 & 29.016 & 29.014 & 29.014 & 0.540 & 0.578 & 0.568 \\
\hline 14 & 29.067 & 29.065 & 29.065 & 0.539 & 0.581 & 0.572 \\
\hline 15 & 29.107 & 29.105 & 29.105 & 0.538 & 0.583 & 0.574 \\
\hline 16 & 29.139 & 29.138 & & 0.538 & 0.585 & \\
\hline 17 & 29.166 & 29.165 & & 0.537 & 0.587 & \\
\hline 18 & 29.189 & 29.187 & & 0.537 & 0.588 & \\
\hline 19 & 29.207 & 29.206 & & 0.537 & 0.589 & \\
\hline 20 & 29.223 & 29.223 & & 0.537 & 0.590 & \\
\hline 21 & 29.237 & 29.236 & & 0.537 & 0.589 & \\
\hline 22 & 29.249 & 29.248 & & 0.537 & 0.589 & \\
\hline 23 & 29.259 & 29.259 & & 0.537 & 0.587 & \\
\hline 24 & 29.268 & 29.268 & & 0.537 & 0.585 & \\
\hline 25 & 29.276 & 29.276 & & 0.538 & 0.581 & \\
\hline 26 & 29.283 & 29.283 & & 0.538 & 0.577 & \\
\hline 27 & 29.289 & 29.289 & & 0.538 & 0.572 & \\
\hline 28 & 29.295 & 29.295 & & 0.538 & 0.566 & \\
\hline 29 & 29.300 & 29.300 & & 0.539 & 0.559 & \\
\hline 30 & 29.304 & 29.304 & & 0.539 & 0.551 & \\
\hline 31 & 29.308 & & & 0.539 & & \\
\hline 32 & 29.312 & & & 0.540 & & \\
\hline 33 & 29.315 & & & 0.540 & & \\
\hline 34 & 29.318 & & & 0.540 & & \\
\hline 35 & 29.321 & & & 0.541 & & \\
\hline 36 & 29.324 & & & 0.541 & & \\
\hline 37 & 29.326 & & & 0.542 & & \\
\hline 38 & 29.328 & & & 0.542 & & \\
\hline 39 & 29.330 & & & 0.542 & & \\
\hline 40 & 29.332 & & & 0.543 & & \\
\hline$\cdots$ & $\cdots$ & $\cdots$ & $\cdots$ & & $\cdots$ & $\cdots$ \\
\hline$\infty$ & 29.367 & 29.367 & 29.367 & & & \\
\hline
\end{tabular}


Table 14. Resonance energies $(E)$ and quantum defect $(\delta)$ of the $3 \mathrm{~s}^{2} 3 \mathrm{p}^{4}\left({ }^{1} \mathrm{D}_{2}\right) n \mathrm{~s}(j=3 / 2$ series originating from the $3 \mathrm{~s}^{2} 3 \mathrm{p}^{5} \mathrm{P}_{3 / 2}^{0}$ ground state of the $\mathrm{Ar}^{+}$ions converging to the $3 \mathrm{~s}^{2} 3 \mathrm{p}^{4}\left({ }^{1} \mathrm{D}_{2}\right)$ threshold of $\mathrm{Ar}^{2+}$. The present results from the Modified atomic orbital theory (MAOT) are compared with the Screening constant by unit nuclear charge (SCUNC) results of Sakho [11] are compared with the QB data of Covington et al., [9]. The energy limits $\mathrm{eV})$ is taken from the NIST tabulations of Ralchenko et al., [17]. $\sigma_{1}\left({ }^{1} \mathrm{D}_{2}\right)=-4.294 \pm$ $0.224 ; \sigma_{2}\left({ }^{1} \mathrm{D}_{2}\right)=16.00$.

\begin{tabular}{|c|c|c|c|c|c|c|}
\hline \multirow{2}{*}{$n$} & MAOT & SCUNC & QB & MAOT & SCUNC & QB \\
\hline & $E$ & $E$ & $E$ & $\delta$ & $\delta$ & $\delta$ \\
\hline 8 & 27.999 & 27.999 & 27.999 & 1.693 & 1.693 & 1.692 \\
\hline 9 & 28.341 & 28.348 & 28.348 & 1.717 & 1.692 & 1.691 \\
\hline 10 & 28.574 & 28.578 & 28.579 & 1.718 & 1.692 & 1.690 \\
\hline 11 & 28.736 & 28.739 & 28.739 & 1.710 & 1.693 & 1.689 \\
\hline 12 & 28.854 & 28.855 & 28.855 & 1.700 & 1.694 & 1.688 \\
\hline 13 & 28.941 & 28.941 & 28.941 & 1.691 & 1.694 & 1.688 \\
\hline 14 & 29.008 & 29.008 & 29.098 & 1.683 & 1.695 & 1.688 \\
\hline 15 & 29.060 & 29.060 & 29.060 & 1.676 & 1.695 & 1.687 \\
\hline 16 & 29.102 & 29.101 & 29.101 & 1.672 & 1.695 & 1.687 \\
\hline 17 & 29.135 & 29.135 & & 1.669 & 1.695 & \\
\hline 18 & 29.163 & 29.162 & & 1.667 & 1.695 & \\
\hline 19 & 29.186 & 29.185 & & 1.667 & 1.694 & \\
\hline 20 & 29.205 & 29.205 & & 1.668 & 1.694 & \\
\hline 21 & 29.221 & 29.221 & & 1.669 & 1.693 & \\
\hline 22 & 29.235 & 29.235 & & 1.672 & 1.692 & \\
\hline 23 & 29.247 & 29.247 & & 1.675 & 1.691 & \\
\hline 24 & 29.258 & 29.258 & & 1.678 & 1.690 & \\
\hline 25 & 29.267 & 29.267 & & 1.682 & 1.689 & \\
\hline 26 & 29.275 & 29.275 & & 1.686 & 1.688 & \\
\hline 27 & 29.282 & 29.282 & & 1.691 & 1.687 & \\
\hline 28 & 29.288 & 29.288 & & 1.695 & 1.686 & \\
\hline 29 & 29.294 & 29.294 & & 1.700 & 1.684 & \\
\hline 30 & 29.299 & 29.299 & & 1.705 & 1.683 & \\
\hline 31 & 29.304 & & & 1.710 & & \\
\hline 32 & 29.308 & & & 1.715 & & \\
\hline 33 & 29.311 & & & 1.720 & & \\
\hline 34 & 29.315 & & & 1.725 & & \\
\hline 35 & 29.318 & & & 1.730 & & \\
\hline 36 & 29.321 & & & 1.735 & & \\
\hline 37 & 29.323 & & & 1.740 & & \\
\hline 38 & 29.326 & & & 1.745 & & \\
\hline 39 & 29.328 & & & 1.750 & & \\
\hline 40 & 29.330 & & & 1.754 & & \\
\hline$\ldots$ & $\ldots$ & $\ldots$ & $\ldots$ & & $\ldots$ & $\ldots$ \\
\hline$\infty$ & 29.367 & 29.367 & 29.367 & & & \\
\hline
\end{tabular}


Table 15. Resonance energies $(E, \mathrm{eV})$ and quantum defect $(\delta)$ of the $3 \mathrm{~s}^{2} 3 \mathrm{p}^{4}\left({ }^{1} \mathrm{D}_{2}\right) n \mathrm{~d}(j=$ $3 / 2$ series originating from the $3 \mathrm{~s}^{2} 3 \mathrm{p}^{52} \mathrm{P}_{3 / 2}^{0}$ ground state of the $\mathrm{Ar}^{+}$ions converging to the $3 s^{2} 3 p^{4}\left({ }^{1} D_{2}\right)$ threshold of $\mathrm{Ar}^{2+}$. The present results from the Modified atomic orbital theory (MAOT) are compared with the Screening constant by unit nuclear charge (SCUNC) results of Sakho [11] are compared with the QB data of Covington et al., [9]. The energy limits eV) is taken from the NIST tabulations of Ralchenko et al., [17]. $\sigma_{1}\left({ }^{1} \mathrm{D}_{2}\right)$ $=-1.575 \pm 0.265 ; \sigma_{2}\left({ }^{1} \mathrm{D}_{2}\right)=16.00$.

\begin{tabular}{|c|c|c|c|c|c|c|}
\hline \multirow{2}{*}{$n$} & MAOT & SCUNC & QB & MAOT & SCUNC & QB \\
\hline & $E$ & $E$ & $E$ & $\delta$ & $\delta$ & $\delta$ \\
\hline 8 & 28.341 & 28.341 & 28.341 & 0.717 & 0.717 & 0.716 \\
\hline 9 & 28.573 & 28.573 & 28.573 & 0.721 & 0.721 & 0.720 \\
\hline 10 & 28.735 & 28.734 & $(28.774)$ & 0.721 & 0.724 & $(0.422)^{*}$ \\
\hline 11 & 28.852 & 28.851 & 28.850 & 0.720 & 0.727 & 0.738 \\
\hline 12 & 28.939 & 28.939 & 28.937 & 0.718 & 0.729 & 0.745 \\
\hline 13 & 29.006 & 29.006 & 29.004 & 0.717 & 0.729 & 0.744 \\
\hline 14 & 29.059 & 29.058 & 29.047 & 0.715 & 0.729 & 0.744 \\
\hline 15 & 29.100 & 29.100 & 29.099 & 0.714 & 0.728 & 0.744 \\
\hline 16 & 29.134 & 29.134 & & 0.713 & 0.727 & \\
\hline 17 & 29.162 & 29.162 & & 0.713 & 0.725 & \\
\hline 18 & 29.185 & 29.185 & & 0.713 & 0.722 & \\
\hline 19 & 29.204 & 29.204 & & 0.712 & 0.720 & \\
\hline 20 & 29.221 & 29.221 & & 0.712 & 0.718 & \\
\hline 21 & 29.235 & 29.235 & & 0.713 & 0.716 & \\
\hline 22 & 29.247 & 29.247 & & 0.713 & 0.714 & \\
\hline 23 & 29.257 & 29.257 & & 0.713 & 0.713 & \\
\hline 24 & 29.267 & 29.267 & & 0.714 & 0.712 & \\
\hline 25 & 29.275 & 29.275 & & 0.714 & 0.712 & \\
\hline 26 & 29.282 & 29.282 & & 0.715 & 0.713 & \\
\hline 27 & 29.288 & 29.288 & & 0.715 & 0.715 & \\
\hline 28 & 29.294 & 29.294 & & 0.716 & 0.719 & \\
\hline 29 & 29.299 & 29.299 & & 0.717 & 0.723 & \\
\hline 30 & 29.304 & 29.303 & & 0.717 & 0.729 & \\
\hline 31 & 29.308 & & & 0.718 & & \\
\hline 32 & 29.311 & & & 0.719 & & \\
\hline 33 & 29.315 & & & 0.719 & & \\
\hline 34 & 29.318 & & & 0.720 & & \\
\hline 35 & 29.321 & & & 0.721 & & \\
\hline 36 & 29.323 & & & 0.722 & & \\
\hline 37 & 29.326 & & & 0.722 & & \\
\hline 38 & 29.328 & & & 0.723 & & \\
\hline 39 & 29.330 & & & 0.724 & & \\
\hline 40 & 29.332 & & & 0.724 & & \\
\hline$\ldots$ & $\ldots$ & $\ldots$ & $\ldots$ & & $\ldots$ & $\ldots$ \\
\hline$\infty$ & 29.367 & 29.367 & 29.367 & & & \\
\hline
\end{tabular}

*This line is not well identified. 
Table 16. Natural widths $(\Gamma, \mathrm{meV})$ of the $\left[3 \mathrm{~s}^{2} 3 \mathrm{p}^{4}\left({ }^{1} \mathrm{D}_{2}\right)\right] n \mathrm{~s}, n \mathrm{~d}(j=1 / 2)$ series originating from the $3 \mathrm{~s}^{2} 3 \mathrm{p}^{52} \mathrm{P}_{1 / 2}^{0}$ metastable state of $\mathrm{Ar}^{+}$ions. The present results from the Modified atomic orbital theory (MAOT) are compared with the Screening constant by unit nuclear charge (SCUNC) results of Sakho [11] are compared with the QB data of Covington et al., [9]. For: $\left[4 \mathrm{~s}^{2} 4 \mathrm{p}^{4}\left({ }^{1} \mathrm{D}_{2}\right)\right] n \mathrm{n}: \sigma_{1}\left({ }^{1} \mathrm{D}_{2}\right)=-5.494 \pm 0.010 ; \sigma_{2}\left({ }^{1} \mathrm{D}_{2}\right)=17.989 \pm 0.010$. For: $\left[4 \mathrm{~s}^{2} 4 \mathrm{p}^{4}\left({ }^{1} \mathrm{D}_{2}\right)\right] n \mathrm{~d}: \sigma_{1}\left({ }^{1} \mathrm{D}_{2}\right)=-0.168 \pm 0.010 ; \sigma_{2}\left({ }^{1} \mathrm{D}_{2}\right)=17.998 \pm 0.010$.

\begin{tabular}{|c|c|c|c|c|c|c|}
\hline \multirow{3}{*}{$n$} & \multicolumn{3}{|c|}{$\left[4 \mathrm{~s}^{2} 4 \mathrm{p}^{4}\left({ }^{1} \mathrm{D}_{2}\right)\right] n \mathrm{~s}$ series } & \multicolumn{3}{|c|}{$\left[4 \mathrm{~s}^{2} 4 \mathrm{p}^{4}\left({ }^{1} \mathrm{D}_{2}\right)\right] n d$ series } \\
\hline & MAOT & SCUNC & QB & MAOT & SCUNC & QB \\
\hline & $\Gamma$ & $\Gamma$ & $\Gamma$ & $\Gamma$ & $\Gamma$ & $\Gamma$ \\
\hline 8 & 103.5 & 103.4 & 103.4 & 0.112 & 0.110 & 0.110 \\
\hline 9 & 64.9 & 64.8 & 64.8 & 0.072 & 0.070 & 0.070 \\
\hline 10 & 43.4 & 43.0 & 43.0 & 0.048 & 0.047 & 0.050 \\
\hline 11 & 30.4 & 30.0 & 30.0 & 0.034 & 0.033 & 0.040 \\
\hline 12 & 22.1 & 21.7 & 21.8 & 0.024 & 0.024 & 0.030 \\
\hline 13 & 16.5 & 16.2 & 16.3 & 0.018 & 0.018 & 0.020 \\
\hline 14 & 12.7 & 12.4 & 12.5 & 0.014 & 0.014 & 0.020 \\
\hline 15 & 9.9 & 9.8 & 9.8 & 0.011 & 0.011 & 0.020 \\
\hline 16 & 7.8 & 7.8 & 7.8 & 0.008 & 0.009 & \\
\hline 17 & 6.3 & 6.4 & & 0.007 & 0.008 & \\
\hline 18 & 5.1 & 5.3 & & 0.005 & 0.006 & \\
\hline 19 & 4.2 & 4.5 & & 0.004 & 0.005 & \\
\hline 20 & 3.5 & 3.8 & & 0.004 & 0.005 & \\
\hline 21 & 2.9 & 3.3 & & 0.003 & 0.004 & \\
\hline 22 & 2.5 & 2.9 & & 0.003 & 0.003 & \\
\hline 23 & 2.1 & 2.5 & & 0.002 & 0.003 & \\
\hline 24 & 1.8 & 2.2 & & 0.002 & 0.003 & \\
\hline 25 & 1.5 & 2.0 & & 0.002 & 0.002 & \\
\hline
\end{tabular}

Table 17. Natural widths $(\Gamma, \mathrm{meV})$ of the $\left[3 \mathrm{~s}^{2} 3 \mathrm{p}^{4}\left({ }^{1} \mathrm{D}_{2}\right)\right] n \mathrm{~s}$, $n \mathrm{~d}(j=3 / 2)$ series originating from the $3 \mathrm{~s}^{2} 3 \mathrm{p}^{52} \mathrm{P}_{1 / 2}^{0}$ metastable state of $\mathrm{Ar}^{+}$ions. The present results from the Modified atomic orbital theory (MAOT) are compared with the Screening constant by unit nuclear charge (SCUNC) results of Sakho [11] are compared with the QB data of Covington et al., [9]. For: $\left[4 \mathrm{~s}^{2} 4 \mathrm{p}^{4}\left({ }^{1} \mathrm{D}_{2}\right)\right] n s: \sigma_{1}\left({ }^{1} \mathrm{D}_{2}\right)=-0.670 \pm 0.010 ; \sigma_{2}\left({ }^{1} \mathrm{D}_{2}\right)=18.038 \pm 0.010$. For: $\left[4 \mathrm{~s}^{2} 4 \mathrm{p}^{4}\right.$ $\left.\left({ }^{1} \mathrm{D}_{2}\right)\right] n \mathrm{~d}: \sigma_{1}\left({ }^{1} \mathrm{D}_{2}\right)=-1.399 \pm 0.010 ; \sigma_{2}\left({ }^{1} \mathrm{D}_{2}\right)=17.827 \pm 0.010$.

\begin{tabular}{ccccccc}
\hline & \multicolumn{3}{c}{$\left[4 \mathrm{~s}^{2} 4 \mathrm{p}^{4}\left({ }^{1} \mathrm{D}_{2}\right)\right] n s$ series } & \multicolumn{3}{c}{$\left[4 \mathrm{~s}^{2} 4 \mathrm{p}^{4}\left({ }^{1} \mathrm{D}_{2}\right)\right] n d$ series } \\
\cline { 2 - 7 }$n$ & MAOT & SCUNC & QB & MAOT & SCUNC & QB \\
\cline { 2 - 7 } & $\Gamma$ & $\Gamma$ & $\Gamma$ & $\Gamma$ & $\Gamma$ & $\Gamma$ \\
\hline 8 & 0.44 & 0.44 & 0.44 & 25.7 & & 25.7 \\
9 & 0.22 & 0.22 & 0.22 & 18.1 & 18.1 \\
10 & 0.13 & 0.11 & 0.13 & 12.9 & & 33.8 \\
11 & 0.08 & 0.06 & 0.08 & 9.3 & & 8.5 \\
\hline
\end{tabular}




\section{Continued}

\begin{tabular}{llllll}
\hline 12 & 0.05 & 0.03 & 0.08 & 6.9 & 3.8 \\
13 & 0.03 & 0.02 & 0.04 & 5.2 & 5.3 \\
14 & 0.02 & 0.01 & 0.02 & 4.0 & 4.4 \\
15 & 0.01 & 0.01 & 0.02 & 3.1 & 3.6 \\
16 & & & & 2.5 & \\
17 & & & & 2.1 & \\
18 & & & 1.7 & \\
19 & & & 1.4 & \\
20 & & & 1.2 & \\
21 & & & 0.9 & \\
22 & & & 0.8 & \\
23 & & & & 0.7 & \\
24 & & & & 0.6 & \\
25 & & & & & \\
\hline
\end{tabular}

Table 18. Resonance energies $(E)$ and quantum defect $(\delta)$ of the $\left[4 s^{2} 4 \mathrm{p}^{4}\left({ }^{1} \mathrm{D}_{2}\right)\right] n d$ series originating from the $4 \mathrm{~s}^{2} 4 \mathrm{p}^{52} \mathrm{P}_{3 / 2}^{\circ}$ ground state of the $\mathrm{Kr}^{+}$ions converging to the $\left[4 \mathrm{~s}^{2} 4 \mathrm{p}^{4}\right.$ $\left({ }^{1} \mathrm{D}_{2}\right)$ ] threshold of $\mathrm{Kr}^{2+}$. The present results from the Modified atomic orbital theory (MAOT) are compared with the Screening constant by unit nuclear charge (SCUNC) results of Sakho [11] are compared with the ALS experimental data of Hinojoha et al., [10]. The ALS resonance energies are calibrated to $\pm 30 \mathrm{meV}$ and quantum defects are estimated to within an error of $20 \%$. The energy limits is taken from the NIST tabulations of Ralchenko et al., [18]. Here $\sigma_{1}\left({ }^{1} \mathrm{D}_{2}\right)=-0.719 \pm 0.116 ; \sigma_{2}\left({ }^{1} \mathrm{D}_{2}\right)=34.00$.

\begin{tabular}{|c|c|c|c|c|c|c|}
\hline \multirow{2}{*}{$n$} & MAOT & SCUNC & ALS & MAOT & SCUNC & ALS \\
\hline & $E$ & $E$ & $E$ & $\delta$ & $\delta$ & $\delta$ \\
\hline 5 & 24.342 & 24.342 & 24.342 & -0.387 & -0.387 & -0.385 \\
\hline 6 & 24.882 & 24.878 & 24.878 & -0.386 & -0.375 & -0.370 \\
\hline 7 & 25.220 & 25.215 & 25.217 & -0.387 & -0.369 & -0.370 \\
\hline 8 & 25.444 & 25.439 & 25.441 & -0.389 & -0.366 & -0.370 \\
\hline 9 & 25.600 & 25.596 & 25.598 & -0.390 & -0.365 & -0.360 \\
\hline 10 & 25.713 & 25.710 & 25.712 & -0.390 & -0.365 & -0.358 \\
\hline 11 & 25.798 & 25.796 & 25.796 & -0.391 & -0.365 & -0.345 \\
\hline 12 & 25.863 & 25.861 & $(25.880)$ & -0.391 & -0.366 & $(-0.671)$ \\
\hline 13 & 25.913 & 25.912 & $(25.926)$ & -0.391 & -0.367 & $(-0.663)$ \\
\hline 14 & 25.954 & 25.953 & & -0.391 & -0.368 & \\
\hline 15 & 25.987 & 25.987 & & -0.390 & -0.369 & \\
\hline 16 & 26.014 & 26.014 & & -0.390 & -0.370 & \\
\hline 17 & 26.037 & 26.037 & & -0.390 & -0.371 & \\
\hline 18 & 26.056 & 26.056 & & -0.389 & -0.372 & \\
\hline 19 & 26.072 & 26.072 & & -0.389 & -0.373 & \\
\hline 20 & 26.086 & 26.086 & & -0.389 & -0.373 & \\
\hline 21 & 26.098 & 26.098 & & -0.388 & -0.374 & \\
\hline 22 & 26.108 & 26.108 & & -0.388 & -0.374 & \\
\hline 23 & 26.118 & 26.117 & & -0.387 & -0.374 & \\
\hline 24 & 26.125 & 26.125 & & -0.387 & -0.374 & \\
\hline
\end{tabular}




\section{Continued}

\begin{tabular}{|c|c|c|c|c|c|}
\hline 25 & 26.133 & 26.132 & & -0.387 & -0.374 \\
\hline 26 & 26.139 & 26.139 & & -0.386 & -0.374 \\
\hline 27 & 26.144 & 26.144 & & -0.386 & -0.374 \\
\hline 28 & 26.149 & 26.149 & & -0.385 & -0.373 \\
\hline 29 & 26.154 & 26.154 & & -0.385 & -0.373 \\
\hline 30 & 26.158 & 26.158 & & -0.385 & -0.372 \\
\hline 31 & 26.162 & & & -0.384 & \\
\hline 32 & 26.165 & & & -0.384 & \\
\hline 33 & 26.168 & & & -0.383 & \\
\hline 34 & 26.171 & & & -0.383 & \\
\hline 35 & 26.174 & & & -0.383 & \\
\hline 36 & 26.176 & & & -0.382 & \\
\hline 37 & 26.178 & & & -0.382 & \\
\hline 38 & 26.180 & & & -0.382 & \\
\hline 39 & 26.182 & & & -0.381 & \\
\hline 40 & 26.184 & & & -0.381 & \\
\hline$\cdots$ & $\ldots$ & $\ldots$ & $\ldots$ & & $\ldots$ \\
\hline$\infty$ & 29.367 & 29.367 & 29.367 & & \\
\hline
\end{tabular}

associated ALS quantum defects are equal to $(-0.671)$ and $(-0.663)$ respectively. For the same levels, the MAOT and SCUNC calculations [11] are respectively at $25.863 \mathrm{eV}$ and $25.861 \mathrm{eV}$ for the $\left[4 \mathrm{~s}^{2} 4 \mathrm{p}^{4}\left({ }^{1} \mathrm{D}_{2}\right)\right] 12 \mathrm{~d}$ state and at $25.913 \mathrm{eV}$ and $25.912 \mathrm{eV}$ for the $\left[4 \mathrm{~s}^{2} 4 \mathrm{p}^{4}\left({ }^{1} \mathrm{D}_{2}\right)\right] 13 \mathrm{~d}$ state. Constant quantum defects are tabulated for the MAOT and SCUNC predictions [11] respectively at -0.31 and -0.37 . So the MAOT and SCUNC estimations can be considered as the accurate data for the $\left[4 \mathrm{~s}^{2} 4 \mathrm{p}^{4}\left({ }^{1} \mathrm{D}_{2}\right)\right] 12 \mathrm{~d}$ and $\left[4 \mathrm{~s}^{2} 4 \mathrm{p}^{4}\left({ }^{1} \mathrm{D}_{2}\right)\right] 13 \mathrm{~d}$ resonances. Table 19 quotes resonance energies and quantum defect of the $\left.4 s^{2} 4 \mathrm{p}^{4}\left({ }^{3} \mathrm{P}_{2}{ }^{3} \mathrm{P}_{1}\right)\right] n s$ and $\left.4 s^{2} 4 \mathrm{p}^{4}\left({ }^{3} \mathrm{P}_{1}\right)\right] n$ series originating from the $4 s^{2} 4 p^{5} \mathrm{P}_{1 / 2}^{0}$ metastable state of the $\mathrm{Kr}$ II ions. For the $\left.4 s^{2} 4 p^{4}\left({ }^{3} \mathrm{P}_{2}\right)\right] n s$ series, comparisons indicate good agreements between theory and experiments. It should be underlined the very good agreements for $n=13-20$. This may enlighten the accuracy of the uncertain ALS measurement [10] listed into parenthesis. For the $\left.4 \mathrm{~s}^{2} 4 \mathrm{p}^{4}\left({ }^{3} \mathrm{P}_{1}\right)\right] n$ series only one ALS data at $23.996 \mathrm{eV}$ is quoted. New MAOT values from $n=14$ to 40 are tabulated with a constant quantum defect about 0.42. In Table 20, resonance energies and quantum defect of the $\left.4 \mathrm{~s}^{2} 4 \mathrm{p}^{4}\left({ }^{3} \mathrm{D}_{2}\right)\right] n \mathrm{~s}$, ndseries originating from the $4 \mathrm{~s}^{2} 4 \mathrm{p}^{5} \mathrm{P}_{1 / 2}^{0}$ metastable state of the $\mathrm{Kr}$ II ions are listed. Here again, the MAOT data agree very well with the ALS data of Hinojoha et al., [10]. It should be underlined the excellent agreements between theory and experiments for the $\left.4 \mathrm{~s}^{2} 4 \mathrm{p}^{4}\left({ }^{3} \mathrm{D}_{2}\right)\right] 8 \mathrm{~s}$ and $\left.4 s^{2} 4 p^{4}\left({ }^{3} D_{2}\right)\right] 9 s$ levels. For these states, both the MAOT and ALS work provide the same values respectively equal to $24.650 \mathrm{eV}$ and $24.842 \mathrm{eV}$. The excellent agreement for the quantum defects can also be mentioned, 0.20 for both theory and experiments. Table 21 presents resonance energies and quantum defect for the 
Table 19. Resonance energies $(E)$ and quantum defect $(\delta)$ of the $\left.4 \mathrm{~s}^{2} 4 \mathrm{p}^{4}\left({ }^{3} \mathrm{P}_{2},{ }^{3} \mathrm{P}_{1}\right)\right] n$ s series originating from the $4 \mathrm{~s}^{2} 4 \mathrm{p}^{52} \mathrm{P}_{1 / 2}^{\circ}$ metastable state of the $\mathrm{Kr}^{+}$ions converging to the $\left[4 s^{2} 4 p^{4}\left({ }^{1} D_{2}\right)\right]$ threshold of $\mathrm{Kr}^{2+}$. The present results from the Modified atomic orbital theory (MAOT) are compared with the Screening constant by unit nuclear charge (SCUNC) results of Sakho [11] are compared with the ALS experimental data of Hinojoha et al., [10]. The ALS resonance energies are calibrated to $\pm 30 \mathrm{meV}$ and quantum defects are estimated to within an error of $20 \%$.The energy limits is taken from the NIST tabulations of Ralchenko et al., [18]. $\sigma_{1}\left({ }^{3} \mathrm{P}_{2}\right)=-1.438 \pm 0.104 ; \sigma_{2}\left({ }^{3} \mathrm{P}_{2}\right)=34.00 ; \sigma_{1}\left({ }^{3} \mathrm{P}_{1}\right)=$ $-0.863 \pm 0.138 ; \sigma_{2}\left({ }^{3} \mathrm{P}_{1}\right)=34.00$.

\begin{tabular}{|c|c|c|c|c|c|c|c|c|}
\hline \multirow{3}{*}{$n$} & \multicolumn{4}{|c|}{$\left.4 \mathrm{~s}^{2} 4 \mathrm{p}^{4}\left({ }^{3} \mathrm{P}_{2}\right)\right] n \mathrm{~s}$} & \multicolumn{4}{|c|}{$\left.4 \mathrm{~s}^{2} 4 \mathrm{p}^{4}\left({ }^{3} \mathrm{P}_{1}\right)\right] n \mathrm{~s}$} \\
\hline & MAOT & ALS & MAOT & ALS & MAOT & ALS & MAOT & ALS \\
\hline & E & $E$ & $\delta$ & $\delta$ & $E$ & $E$ & $\delta$ & $\delta$ \\
\hline 11 & 23.910 & $(23.906)$ & -0.771 & $(-0.716)$ & & & & \\
\hline 12 & 23.969 & 23.969 & -0.765 & -0.779 & & & & \\
\hline 13 & 24.016 & 24.016 & -0.762 & -0.791 & & & & \\
\hline 14 & 24.053 & $(24.059)$ & -0.761 & $(-0.940)$ & 23.957 & & 0.418 & \\
\hline 15 & 24.084 & 24.083 & -0.761 & -0.730 & 23.996 & 23.996 & 0.419 & 0.403 \\
\hline 16 & 24.109 & 24.109 & -0.762 & -0.774 & 24.028 & & 0.420 & \\
\hline 17 & 24.131 & $(24.129)$ & -0.762 & $(-0.686)$ & 24.054 & & 0.420 & \\
\hline 18 & 24.148 & $(24.147)$ & -0.763 & $(-0.707)$ & 24.076 & & 0.420 & \\
\hline 19 & 24.164 & $(24.162)$ & -0.764 & $(-0.659)$ & 24.094 & & 0.420 & \\
\hline 20 & 24.177 & $(24.176)$ & -0.764 & $(-0.731)$ & 24.110 & & 0.420 & \\
\hline 21 & 24.188 & & -0.765 & & 24.124 & & 0.420 & \\
\hline 22 & 24.198 & & -0.765 & & 24.135 & & 0.420 & \\
\hline 23 & 24.207 & & -0.765 & & 24.145 & & 0.419 & \\
\hline 24 & 24.214 & & -0.766 & & 24.154 & & 0.419 & \\
\hline 25 & 24.221 & & -0.766 & & 24.162 & & 0.419 & \\
\hline 26 & 24.227 & & -0.766 & & 24.169 & & 0.419 & \\
\hline 27 & 24.232 & & -0.766 & & 24.175 & & 0.419 & \\
\hline 28 & 24.237 & & -0.766 & & 24.180 & & 0.419 & \\
\hline 29 & 24.242 & & -0.766 & & 24.185 & & 0.418 & \\
\hline 30 & 24.246 & & -0.766 & & 24.190 & & 0.418 & \\
\hline 31 & 24.249 & & -0.766 & & 24.194 & & 0.418 & \\
\hline 32 & 24.252 & & -0.765 & & 24.197 & & 0.418 & \\
\hline 33 & 24.255 & & -0.765 & & 24.201 & & 0.418 & \\
\hline 34 & 24.258 & & -0.765 & & 24.204 & & 0.418 & \\
\hline 35 & 24.260 & & -0.765 & & 24.206 & & 0.418 & \\
\hline 36 & 24.263 & & -0.765 & & 24.209 & & 0.418 & \\
\hline 37 & 24.265 & & -0.764 & & 24.211 & & 0.418 & \\
\hline 38 & 24.267 & & -0.764 & & 24.213 & & 0.418 & \\
\hline 39 & 24.269 & & -0.764 & & 24.215 & & 0.418 & \\
\hline 40 & 24.270 & & -0.763 & & 24.217 & & 0.418 & \\
\hline$\cdots$ & & & & & & & & \\
\hline$\infty$ & 24.303 & 24.303 & & & 24.252 & 24.252 & & \\
\hline
\end{tabular}


Table 20. Resonance energies $(E)$ and quantum defect $(\delta)$ of the $\left.4 \mathrm{~s}^{2} 4 \mathrm{p}^{4}\left({ }^{3} \mathrm{D}_{2}\right)\right] n s$, $n$ d series originating from the $4 \mathrm{~s}^{2} 4 \mathrm{p}^{52} \mathrm{P}_{1 / 2}^{\circ}$ metastable state of the $\mathrm{Kr}^{+}$ions converging to the $\left[4 \mathrm{~s}^{2} 4 \mathrm{p}^{4}\left({ }^{1} \mathrm{D}_{2}\right)\right]$ threshold of $\mathrm{Kr}^{2+}$. The present results from the Modified atomic orbital theory (MAOT) are compared with the Screening constant by unit nuclear charge (SCUNC) results of Sakho [11] are compared with the ALS experimental data of Hinojoha et al., [10]. The ALS resonance energies are calibrated to $\pm 30 \mathrm{meV}$ and quantum defects are estimated to within an error of $20 \%$. The energy limits is taken from the NIST tabulations of Ralchenko et al., [18]. $\sigma_{1}\left({ }^{1} \mathrm{D}_{2}\right)=-0.414 \pm 0.116 ; \sigma_{2}\left({ }^{1} \mathrm{D}_{2}\right)=34.00$ for $\left.4 \mathrm{~s}^{2} 4 \mathrm{p}^{4}\left({ }^{3} \mathrm{D}_{2}\right)\right]$ ns. $\sigma_{1}\left({ }^{1} \mathrm{D}_{2}\right)=-0.698 \pm 0.116 ; \sigma_{2}\left({ }^{1} \mathrm{D}_{2}\right)=34.00$ for $\left.4 \mathrm{~s}^{2} 4 \mathrm{p}^{4}\left({ }^{3} \mathrm{D}_{2}\right)\right] n \mathrm{~d}$.

\begin{tabular}{|c|c|c|c|c|c|c|c|c|}
\hline \multirow{3}{*}{$n$} & \multicolumn{4}{|c|}{$\left.4 \mathrm{~s}^{2} 4 \mathrm{p}^{4}\left({ }^{3} \mathrm{D}_{2}\right)\right] n \mathrm{~s}$} & \multicolumn{4}{|c|}{$\left.4 \mathrm{~s}^{2} 4 \mathrm{p}^{4}\left({ }^{3} \mathrm{D}_{2}\right)\right] n \mathrm{~d}$} \\
\hline & MAOT & ALS & MAOT & ALS & MAOT & ALS & MAOT & ALS \\
\hline & $E$ & $E$ & $\delta$ & $\delta$ & $E$ & $E$ & $\delta$ & $\delta$ \\
\hline 6 & 23.927 & 23.927 & 0.200 & 0.200 & 24.214 & 24.214 & -0.371 & -0.375 \\
\hline 7 & 24.368 & $(24.366)$ & 0.200 & $(0.205)$ & 24.553 & 24.551 & -0.369 & -0.371 \\
\hline 8 & 24.650 & 24.650 & 0.200 & $0.204)$ & 24.778 & 24.775 & -0.370 & -0.371 \\
\hline 9 & 24.842 & 24.842 & 0.200 & 0.201 & 24.935 & 24.933 & -0.371 & -0.375 \\
\hline 10 & 24.978 & - & 0.199 & - & 25.049 & 25.047 & -0.372 & -0.379 \\
\hline 11 & 25.079 & 25.082 & 0.199 & 0.152 & 25.134 & 25.132 & -0.372 & -0.363 \\
\hline 12 & 25.154 & 25.158 & 0.199 & 0.139 & 25.199 & 25.196 & -0.373 & -0.349 \\
\hline 13 & 25.213 & & 0.198 & & 25.251 & 25.248 & -0.373 & -0.364 \\
\hline 14 & 25.259 & & 0.198 & & 25.292 & & -0.373 & \\
\hline 15 & 25.297 & & 0.198 & & 25.325 & & -0.373 & \\
\hline 16 & 25.327 & & 0.198 & & 25.352 & & -0.373 & \\
\hline 17 & 25.352 & & 0.198 & & 25.375 & & -0.373 & \\
\hline 18 & 25.373 & & 0.198 & & 25.394 & & -0.373 & \\
\hline 19 & 25.391 & & 0.198 & & 25.410 & & -0.373 & \\
\hline 20 & 25.406 & & 0.198 & & 25.424 & & -0.373 & \\
\hline 21 & 25.419 & & 0.198 & & 25.436 & & -0.373 & \\
\hline 22 & 25.431 & & 0.198 & & 25.446 & & -0.372 & \\
\hline 23 & 25.440 & & 0.198 & & 25.455 & & -0.372 & \\
\hline 24 & 25.449 & & 0.198 & & 25.463 & & -0.372 & \\
\hline 25 & 25.457 & & 0.198 & & 25.470 & & -0.372 & \\
\hline 26 & 25.463 & & 0.198 & & 25.477 & & -0.371 & \\
\hline 27 & 25.469 & & 0.198 & & 25.482 & & -0.371 & \\
\hline 28 & 25.475 & & 0.198 & & 25.487 & & -0.371 & \\
\hline 29 & 25.479 & & 0.198 & & 25.492 & & -0.371 & \\
\hline 30 & 25.484 & & 0.198 & & 25.496 & & -0.370 & \\
\hline 31 & 25.488 & & 0.198 & & 25.500 & & -0.370 & \\
\hline 32 & 25.491 & & 0.198 & & 25.503 & & -0.370 & \\
\hline 33 & 25.494 & & 0.198 & & 25.506 & & -0.370 & \\
\hline 34 & 25.497 & & 0.198 & & 25.509 & & -0.369 & \\
\hline 35 & 25.500 & & 0.198 & & 25.511 & & -0.369 & \\
\hline 36 & 25.503 & & 0.198 & & 25.514 & & -0.369 & \\
\hline 37 & 25.505 & & 0.199 & & 25.516 & & -0.369 & \\
\hline 38 & 25.507 & & 0.199 & & 25.518 & & -0.368 & \\
\hline 39 & 25.509 & & 0.199 & & 25.520 & & -0.368 & \\
\hline 40 & 25.511 & & 0.199 & & 25.522 & & -0.368 & \\
\hline$\ldots$ & & & & & & & & \\
\hline$\infty$ & 25.545 & 25.545 & & & 25.555 & 25.555 & & \\
\hline
\end{tabular}


Table 21. Resonance energies $(E)$ and quantum defect $(\delta)$ of the $\left.4 \mathrm{~s}^{2} 4 \mathrm{p}^{4}\left({ }^{3} \mathrm{D}_{2},{ }^{1} \mathrm{~S}_{0}\right)\right] n s$, $n \mathrm{~d}$ series of the $\mathrm{Kr}^{+}$ions. The present results from the Modified atomic orbital theory (MAOT) are compared with the Screening constant by unit nuclear charge (SCUNC) results of Sakho [11] are compared with the ALS experimental data of Hinojoha et al., [10]. The ALS resonance energies are calibrated to $\pm 30 \mathrm{meV}$ and quantum defects are estimated to within an error of $20 \% . \sigma_{1}\left({ }^{1} \mathrm{D}_{2}\right)=-0.785 \pm 0.116 ; \sigma_{2}\left({ }^{1} \mathrm{D}_{2}\right)=34.00 ; \sigma_{1}\left({ }^{1} \mathrm{~S}_{0}\right)=$ $-0.811 \pm 0.116 ; \sigma_{2}\left({ }^{1} \mathrm{~S}_{0}\right)=34.00$.

\begin{tabular}{|c|c|c|c|c|c|c|c|c|}
\hline \multirow{3}{*}{$n$} & \multicolumn{4}{|c|}{$4 \mathrm{~s}^{2} 4 \mathrm{p}^{52} \mathrm{P}_{1 / 2}^{0} \rightarrow 4 \mathrm{~s}^{2} 4 \mathrm{p}^{4}\left({ }^{1} \mathrm{~S}_{0}\right] n \mathrm{~s}$} & \multicolumn{4}{|c|}{$\left.4 \mathrm{~s}^{2} 4 \mathrm{p}^{5}{ }^{2} \mathrm{P}_{3 / 2}^{\circ} \rightarrow 4 \mathrm{~s}^{2} 4 \mathrm{p}^{4}\left({ }^{1} \mathrm{~S}_{0}\right)\right] n d$} \\
\hline & MAOT & ALS & MAOT & ALS & MAOT & ALS & MAOT & ALS \\
\hline & $E$ & $E$ & $\delta$ & $\delta$ & $E$ & $E$ & $\delta$ & $\delta$ \\
\hline 4 & 23.738 & 23.738 & 0.357 & 0.358 & & & & \\
\hline 5 & 25.315 & 25.312 & 0.358 & 0.360 & 25.965 & $(25.947)$ & 0.369 & $(0.385)$ \\
\hline 6 & 26.132 & 26.124 & 0.355 & 0.368 & 26.780 & 26.780 & 0.380 & 0.379 \\
\hline 7 & 26.608 & 26.602 & 0.353 & 0.371 & 27.261 & $(27.261)$ & 0.381 & $(0.379)$ \\
\hline 8 & 26.910 & 26.904 & 0.352 & 0.376 & 27.566 & 27.566 & 0.381 & 0.376 \\
\hline 9 & 27.112 & 27.108 & 0.351 & 0.377 & 27.771 & 27.771 & 0.379 & 0.378 \\
\hline 10 & 27.255 & $(27.261)$ & 0.351 & $(0.303)$ & 27.915 & 27.914 & 0.378 & 0.381 \\
\hline 11 & 27.360 & 27.356 & 0.351 & 0.400 & 28.021 & 28.020 & 0.378 & 0.383 \\
\hline 12 & 27.439 & 27.435 & 0.351 & 0.412 & 28.100 & 28.099 & 0.377 & 0.381 \\
\hline 13 & 27.500 & (27.495) & 0.351 & $(0.435)$ & 28.161 & $(28.160)$ & 0.377 & $(0.394)$ \\
\hline 14 & 27.548 & $(27.544)$ & 0.352 & $(0436)$ & 28.210 & $(28.208)$ & 0.376 & $(0.394)$ \\
\hline 15 & 27.586 & & 0.352 & & 28.249 & $(28.247)$ & 0.376 & $(0.418)$ \\
\hline 16 & 27.618 & & 0.353 & & 28.280 & & 0.376 & \\
\hline 17 & 27.644 & & 0.354 & & 28.306 & & 0.376 & \\
\hline 18 & 27.665 & & 0.354 & & 28.328 & & 0.376 & \\
\hline 19 & 27.683 & & 0.355 & & 28.346 & & 0.376 & \\
\hline 20 & 27.699 & & 0.356 & & 28.362 & & 0.376 & \\
\hline 21 & 27.712 & & 0.356 & & 28.375 & & 0.377 & \\
\hline 22 & 27.724 & & 0.357 & & 28.387 & & 0.377 & \\
\hline 23 & 27.734 & & 0.358 & & 28.397 & & 0.377 & \\
\hline 24 & 27.743 & & 0.358 & & 28.405 & & 0.377 & \\
\hline 25 & 27.750 & & 0.359 & & 28.413 & & 0.378 & \\
\hline 26 & 27.757 & & 0.359 & & 28.420 & & 0.378 & \\
\hline 27 & 27.763 & & 0.360 & & 28.426 & & 0.378 & \\
\hline 28 & 27.769 & & 0.361 & & 28.432 & & 0.379 & \\
\hline 29 & 27.774 & & 0.361 & & 28.437 & & 0.379 & \\
\hline 30 & 27.778 & & 0.362 & & 28.441 & & 0.379 & \\
\hline 31 & 27.782 & & 0.362 & & 28.445 & & 0.379 & \\
\hline 32 & 27.786 & & 0.363 & & 28.449 & & 0.380 & \\
\hline 33 & 27.789 & & 0.363 & & 28.452 & & 0.380 & \\
\hline 34 & 27.792 & & 0.364 & & 28.455 & & 0.380 & \\
\hline 35 & 27.795 & & 0.364 & & 28.458 & & 0.381 & \\
\hline 36 & 27.797 & & 0.365 & & 28.460 & & 0.381 & \\
\hline 37 & 27.799 & & 0.365 & & 28.462 & & 0.381 & \\
\hline 38 & 27.802 & & 0.366 & & 28.465 & & 0.382 & \\
\hline 39 & 27.804 & & 0.366 & & 28.467 & & 0.382 & \\
\hline 40 & 27.805 & & 0.366 & & 28.468 & & 0.382 & \\
\hline$\cdots$ & & & & & & & & \\
\hline$\infty$ & 27.840 & 27.840 & & & 28.503 & 28.503 & & \\
\hline
\end{tabular}


$\left.4 \mathrm{~s}^{2} 4 \mathrm{p}^{4}\left({ }^{3} \mathrm{D}_{2},{ }^{1} \mathrm{~S}_{0}\right)\right] n s, n d$ series of the Kr II ions. Here again, the good agreements between the present MAOT and ALS data [10] may enlighten the accuracy of the uncertain ALS measurement listed into parenthesis. Overall, for both the $\mathrm{Cl}$ II, Ar II and $\mathrm{Kr}$ II ions, it is demonstrated in this paper the possibilities to reproduce excellently high ALS measurements from single MAOT analytical expression. This is the main strength of the present work.

\section{Conclusion}

Accurate high lying resonance energy up to $n=40$ is reported applying the Modified atomic orbital theory. For both the Cl II, Ar II and Kr II ions investigated, a single formula has been established to reproduce with a very good accuracy high experimental measurements such as those performed at the Advanced Light Source at Lawrence Berkeley National Laboratory. A huge number of results are tabulated as useful reference data for interpreting atomic spectra from astrophysical objects containing chlorine, argon and krypton elements.

\section{Conflicts of Interest}

The authors declare no conflicts of interest regarding the publication of this paper.

\section{References}

[1] Garnett, D.R. (1989) The Astrophysical Journal, 345, 771.

[2] Küppers, M.E. and Schneider, N.M. (2000) Geophysical Research Letters, 25, 513-516. https://doi.org/10.1029/1999GL010718

[3] Keenan, et al. (2003) The Astrophysical Journal, 543, 385.

[4] Hernández, E.M., Juárez, A.M., Kilcoyne, A.L.D., Aguilar, A., Hernández, L., Antillón, A., et al. (2015) Journal of Quantitative Spectroscopy and Radiative Transfer, 151, 217-223. https://doi.org/10.1016/j.jqsrt.2014.10.004

[5] McLaughlin, B.M. (2017) Monthly Notices of the Royal Astronomical Society, 464, 1990-1999. https://doi.org/10.1093/mnras/stw2520

[6] Lewis,W. (2005) Chandra Proposal ID\# 07500185.

[7] Kraus, M., Borges Fernandes, M., de Araújo, F.X. and Lamers, H.J.G.L.M. (2005) Astronomy and Astrophysics, 441, 289-302.

https://doi.org/10.1051/0004-6361:20047013

[8] Kniazev, A.Y., Grebel, E.K., Pustilnik, S.A., Pramskij, A.G. and Zucker, D.B. (2005) The Astronomical Journal, 130,1558-1573. https://doi.org/10.1086/432931

[9] Covington, A.M., Aguilar, A., Covington, I.R., Hinojosa, G., Shirley, C.A., Phaneuf, R.A., et al. (2011) Physical Review A, 84, 013413. https://doi.org/10.1103/PhysRevA.84.013413

[10] Hinojoha, G., Covington, A.M., Alna'Washi, G.A., Lu, M., Phaneuf, R.A., Sant'Anna, M.M., et al. (2012) Physical Review A, 86, 063402.

https://doi.org/10.1103/PhysRevA.86.063402

[11] Sakho, I. (2018) The Screening Constant by Unit Nuclear Charge Method, Description \& Application to the Photoionization of Atomic Systems. ISTE Science Publishing Ltd, London and John Wiley \& Sons, Inc. USA. 
https://doi.org/10.1002/9781119476948

[12] Sakho, I. (2013) Chinese Journal of Physics, 51, 2.

[13] Sakho, I. (2014) Journal of Atomic and Molecular Science, 5, 206.

[14] Sakho, I. (2014) Journal of Atomic and Molecular Science, 5, 206.

[15] Diop, B., et al. (2014) Chinese Journal of Physics, 52, 1223.

[16] Sow, M., et al. (2014) Chinese Journal of Physics, 52, 1459.

[17] Ralchenko, Y., Kramida, A.E. and Reader, J. (2010) NIST Atomic Spectra Database, (Version 4.0.1). National Institute of Standards and Technology, Gaithersburg.

[18] Ralchenko, Y., Kramida, A.E. and Reader, J. (2011) NIST Atomic Spectra Database (Version 4.0.1). National Institute of Standards, Technology, Gaithersburg, MD.

[19] Ralchenko, Y., Kramida, A.E. and Reader, J. (2014) NIST Atomic Spectra Database (Version 5.2). National Institute of Standards, Technology, Gaithersburg, MD. 